\title{
Chemical abundances of hot post-AGB stars ${ }^{\star}$
}

\author{
D. R. C. Mello ${ }^{1}$, S. Daflon ${ }^{1}$, C. B. Pereira ${ }^{1}$, and I. Hubeny ${ }^{2}$ \\ ${ }^{1}$ Observatório Nacional/MCT, Rua Gen. José Cristino 77, CEP20921400, São Cristovão, Rio de Janeiro, Brazil \\ e-mail: [mello; daflon; claudio]@on.br \\ 2 Steward Observatory and Department of Astronomy, University of Arizona, Tucson, AZ 85721, USA \\ e-mail: hubeny@as.arizona.edu
}

Received 28 June 2011 / Accepted 27 March 2012

\section{ABSTRACT}

\begin{abstract}
Context. Hot post-AGB stars are luminous objects of low- and intermediate mass $\left(0.8-8 M_{\odot}\right)$ in the final stage of evolution, moving between the asymptotic giant branch (AGB) stars and planetary nebulae. The absorption lines observed in their spectra are typical of early-type stars and their abundance pattern may be associated with the occurence of one or more mixing processes during the previous AGB phase.

Aims. To better constrain their observed chemical pattern and evolutionary status, we determined chemical abundances for a sample of hot post-AGB stars selected according to spectroscopic criteria.

Methods. The observational data are high-resolution spectra obtained with the FEROS spectrograph. The stellar parameters and chemical composition were obtained from fully consistent non-LTE synthesis.

Results. The general abundance pattern reveals relevant nitrogen enrichment, slight depletion in carbon and sulfur and mild excess in helium for most of the objects. One notable exception is LSE 148, with $Z=0.001$, which is likely to be a metal-poor object at high galactic latitude. The atmospheric parameters and chemical abundances obtained are discussed in the context of evolutionary models. Mixing processes like the second/third dredged-up and "hot bottom-burning" are invoked to explain the obtained results.
\end{abstract}

Key words. stars: abundances - stars: AGB and post-AGB - stars: atmospheres - stars: evolution - stars: fundamental parameters stars: early-type

\section{Introduction}

Post-asymptotic giant branch (post-AGB, hereafter) stars are luminous objects of low and intermediate-mass $\left(0.8-8 M_{\odot}\right)$ in the final stage of evolution. At the end of the AGB phase they suffer very strong mass loss and evolve rapidly to higher temperatures at rougly constant luminosity. However, they are not hot enough to ionize the circumstellar envelope and emerge as planetary nebulae (PNe). Post-AGB objects have typical luminosities $\sim 10^{4} L_{\odot}$ and the transition time to PNe strongly depends on the initial mass, which varies from about 30 years for the most massive objects to 30000 years for the less massive ones (Schönberner 1979a,b, 1983). Because the transition time is short, post-AGB objects are rare.

Another important characteristic of the post-AGB stars is the presence of a thick circumstelar envelope, developed during the thermally pulsing AGB phase. Stars evolve into the post-AGB phase and appear in the optical as a consequence of the dilution of the circumstellar envelope on a timescale that is dependent on the initial mass of the progenitor star (Blöcker 1995).

Many papers devoted to post-AGB stars point out some open questions that still deserve closer investigation. For example, Kwok (2000) quotes the mechanism of the PN formation in the transition from post-AGB to PNe. Cerrigone et al. (2009) quotes the dual-dust chemistry phenomenon observed not only in the transition objects but also during the PN stage. Herwig (2005) mentions the ocurring mechanisms on the AGB nucleosynthesis and the mixing processes, such as dredged-up

* Based on observations made with the $2.2 \mathrm{~m}$ telescope at the European Southern Observatory (La Silla, Chile). and hot-bottom-burning. The post-AGB properties have been reviewed by Hrivnak (1997), Van Winckel (2003), and Waelkens \& Waters (2004).

The so-called optically bright post-AGB stars are often late-type stars, with spectral types F-G. However, some postAGB stars present absorption profiles that resemble those of typical OB stars. They are denominated hot post-AGB stars. Several surveys aiming to characterize the nature of early-type stars observed at the high galactic latitude have found an impressive number of stars that are apparently evolved (Greenstein \& Sargent 1974; Conlon et al. 1992; Magee et al. 2001; Martin 2004). Most of these targets have been classified as posthorizontal branch stars (PHB) or blue-horizontal branch stars (BHB) with a significant number of them being classified as hot post-AGB stars (McCausland et al. 1992).

An interesting characteristic observed in some hot postAGB stars is that their very short evolutionary timescales allow us to observe intrinsic changes during this final phase of stellar evolution. A known case is the hot post-AGB object SAO 244567, which displayed spectral changes in the past decades and seems to be rapidly evolving to PN stage (Parthasarathy et al. 1993). On the other hand, some objects are related with the "born-again" phenomenon e.g. the objects V4334 Sgr, FG Sge and V605 Aql which have evolved following a late thermal pulse from a white dwarf phase to a post-AGB phase again (Van Winckel 2003).

In the past two decades several groups analysed this class of post-AGB stars based on different techniques. Some papers reported modern surveys to detect new hot post-AGB stars and candidates (Oudmaijer 1996). Some works focused on lowresolution spectra of hot post-AGB stars to identify important 
spectroscopic characteristics and make a preliminary classification of these objects (Parthasarathy et al. 2000). Chemical analyses based on high-resolution spectra have been presented, for example, by McCausland et al. (1992), Venn et al. (1998), Ryans et al. (2003), and Sarkar et al. (2005).

Post-AGB stars show a wide variety of chemical patterns. For example, carbon-rich as well as apparently carbon-defficient (Reyniers et al. 2007; Moehler \& Heber 1998) objects have been identified. Several peculiar characteristics have been detected by some authors. One of them is the carbon deficiency that is possibly related to the non-occurrence of the third dredge-up episode in the previous AGB phase or the activation of "hot bottomburning" (hereafter HBB) process. Additionally, some stars may display higher nitrogen abundances while the $s$-process elements may be absent. These questions related to the abundance pattern in hot post-AGB stars have still being discussed to understand the origins of classical post-AGB and hot post-AGB stars (Van Winckel 2003).

Motivated by the questions mentioned above, we obtained elemental abundances for a sample of hot post-AGB stars to better constrain the abundance pattern of these objects. Our analysis is based on non-LTE synthetic spectra fitted to high-resolution observed profiles. This paper is organized as follows: in Sect. 2 we describe the selection criteria of the sample and present the instrumental set-up for the high-resolution spectra of the objects. In Sect. 3 the complete analysis of the sample is presented (spectral characteristics, applied methodology, obtained results for the atmospheric parameters, and chemical abundances) and in Sect. 4 we dicuss the results and implications of the evolutionary status for the objects.

\section{Observations}

Our sample contains ten hot post-AGB stars selected from Parthasarathy et al. (2000), based on low-resolution spectra, and the Torun catalogue (Szczerba et al. 2007, 2011), a recent compilation of post-AGB stars and candidates. Additional references were used to provide complete information about the sample, such as the southern LS, LSS and LSE stars catalogue (Drilling \& Bergeron 1995), the low-resolution spectroscopic atlas of post-AGB stars and related objects (Suarez et al. 2006) and the compilation of post-AGB objects of Stasińska et al. (2006).

The selected objects were placed in the IRAS colour-colour diagram. In this diagram, the infrared colours are defined as usual (Van der Veen \& Habing 1988),

$$
\begin{aligned}
& {[12]-[25]=-2.5 \log \frac{F_{12}}{F_{25}}} \\
& {[25]-[60]=-2.5 \log \frac{F_{25}}{F_{60}}}
\end{aligned}
$$

where $F_{12}, F_{25}$ and $F_{60}$ are the fluxes measured at 12,25 , and $60 \mu \mathrm{m}$. The PNe and post-AGB stars have a specific locus in the IRAS diagram, as can be seen in Fig. 1, where we present the location of all objects of our sample plus a sample of Galactic PNe (Perinotto et al. 2004) for comparison. These objects are expected to have IRAS colours within the box of $F(12 \mu \mathrm{m}) / F(25 \mu \mathrm{m}) \leq 0.50$ and $F(25 \mu \mathrm{m}) / F(60 \mu \mathrm{m}) \geq 0.3$ (Pottasch et al. 1988).

Our analysis is based on high-resolution spectra obtained at the $2.2 \mathrm{~m}$ MPI telescope at ESO (La Silla, Chile) with the FEROS (Fiber-fed Extended Range Optical Spectrograph) echelle spectrograph (Kaufer et al. 1999). The spectral resolution

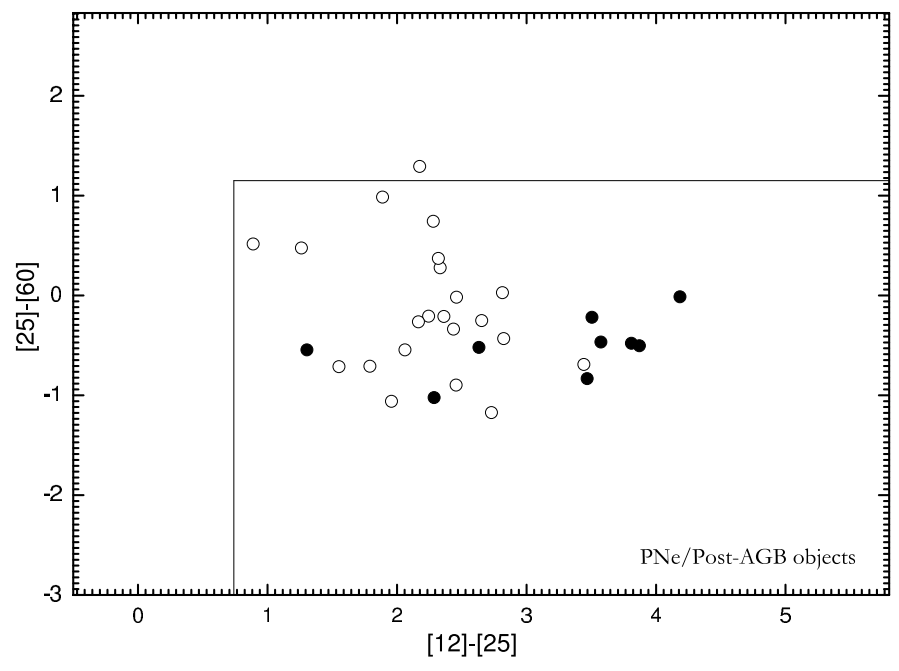

Fig. 1. IRAS colour-colour diagram showing the location of all the objects of our sample (filled circles), except for LSE 148, which has no measurements of IRAS fluxes. Solid lines shows the post-AGB/PNe locus defined by Pottasch et al. (1988). Open circles represent the sample of Galactic PNe of Perinotto et al. (2004) for comparison.

is $R=48000$ corresponding to 2.2 pixels of $15 \mu \mathrm{m}$ and the wavelenght coverage is from $3600 \AA$ to $9200 \AA$ in 39 orders. The nominal signal-to-noise ratio $(\mathrm{S} / \mathrm{N})$ is determined by measurement of the rms flux fluctuation in selected continuum slots, and a typical value obtained is $S / N \sim 100-150$ for all observed objects. The spectra were obtained in two observing runs in September 2007 and May 2008 under the ESO/ON agreement. The data reduction followed a standard procedure, using the FEROS pipeline. Spectral types, $V$ magnitudes, observation dates and IRAS fluxes of the targets are shown in Table 1. The object designations were taken from the SIMBAD ${ }^{1}$ database along with the spectral classification and $V$ magnitudes. The Table 1 also contains our measurements of heliocentric radial velocities $V_{r}$ for all objects in the sample, except for SAO 209306 (more details in Sect. 3.1.1). Figure 2 shows examples of observed spectra in the region 4600-4700 $\AA$ for all targets, normalized to a unit continuum and vertically shifted for clarity.

\section{Analysis}

\subsection{Description of the spectra}

The high-resolution spectra of the targets show many absorp-

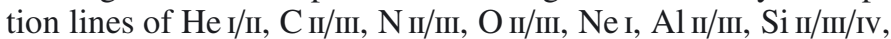
$\mathrm{Mg}$ II, and $\mathrm{S}_{\text {II }} / \mathrm{III}$. All objects show rich emission-line spectra, except for the stars LSE 148 and SAO 209306, where no emission lines are detected. Emission lines caused by $\mathrm{C}_{\text {III }}$, O I, Fe II/III, Si II, $\mathrm{Mn}_{\mathrm{I}}, \mathrm{V}_{\mathrm{I}}$ and the forbidden emission lines of [Fe II/III], [O I/II], [N II], [S II] and [Ni II] have been detected for many objects. The detection of low-excitation nebular emission lines caused by [O II], [ $\left.\mathrm{N}_{\mathrm{II}}\right]$ and [S II] can be associated with the proto-planetary nebulae nature for some objects in our sample. This fact will be discussed on Sect. 4.2.

Some objects show P-Cyg profiles of $\mathrm{H}_{\mathrm{I}}, \mathrm{He}_{\mathrm{I}}, \mathrm{C}_{\text {II, }}$, and Fe III in their spectra. These P-Cyg profiles suggests an ongoing massloss process in these objects. Other interesting charateristics detected in the spectra of hot post-AGB are the NaI D and O I triplet at $\sim 7773 \AA$ with some line components in emission.

${ }^{1}$ http://simbad.u-strasbg.fr/simbad/ 
D. R. C. Mello et al.: Chemical abundances of hot post-AGB stars
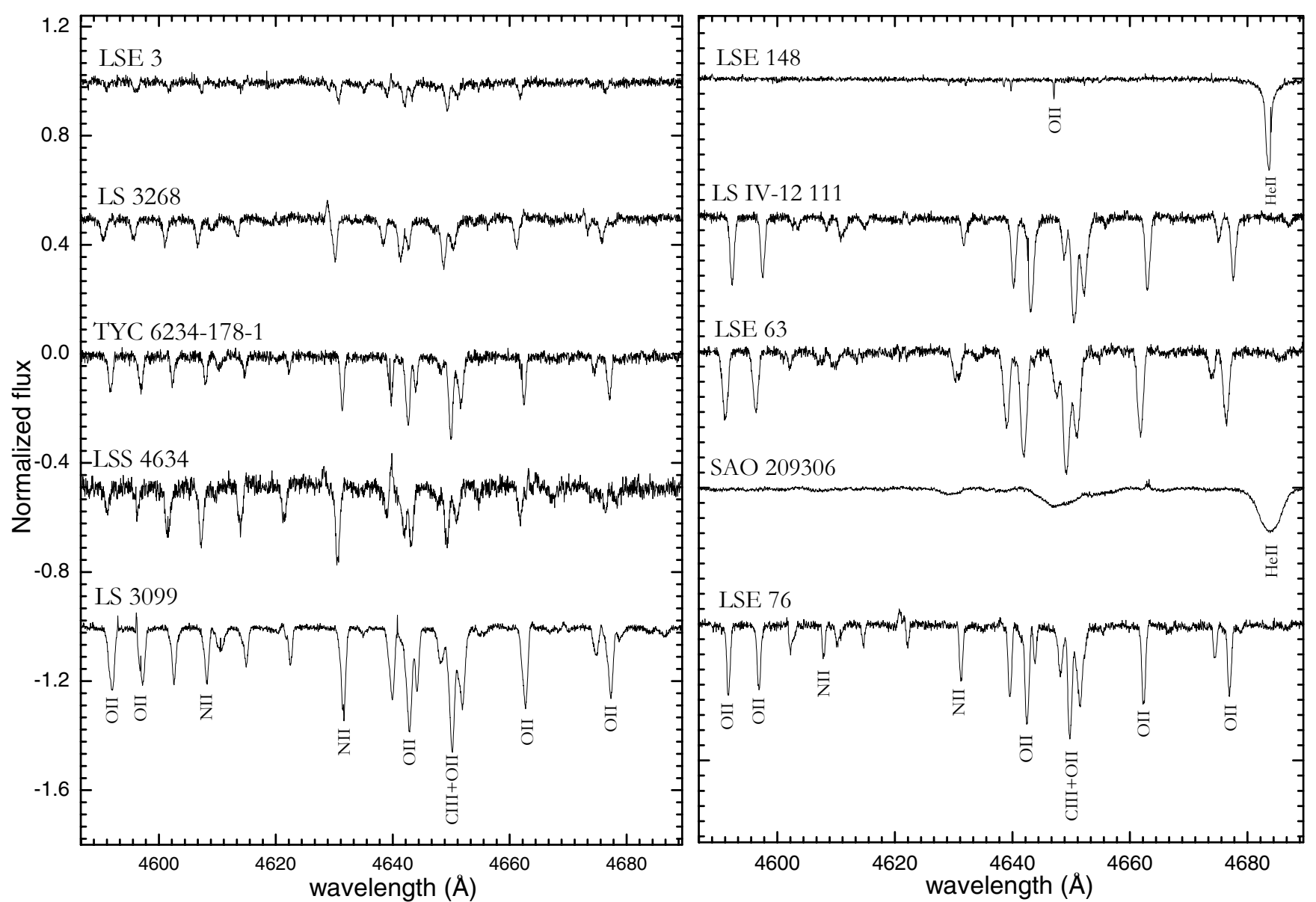

Fig. 2. Examples of normalized spectra of our sample objects in the region 4600-4700 A with the identification of the some of the main spectral lines in this interval.

Table 1. Observational data.

\begin{tabular}{|c|c|c|c|c|c|c|c|c|c|}
\hline \multirow{2}{*}{$\begin{array}{l}\text { Object } \\
\text { (Name) }\end{array}$} & \multirow[t]{2}{*}{ IRAS } & \multirow[t]{2}{*}{ MK } & \multirow{2}{*}{$\begin{array}{c}V \\
(\mathrm{mag})\end{array}$} & \multirow{2}{*}{$\begin{array}{l}\text { Mean } V_{r} \\
\left(\mathrm{~km} \mathrm{~s}^{-1}\right)\end{array}$} & \multirow[t]{2}{*}{ Date } & \multicolumn{4}{|c|}{ IRAS fluxes (Jy) } \\
\hline & & & & & & $12 \mu \mathrm{m}$ & $25 \mu \mathrm{m}$ & $60 \mu \mathrm{m}$ & $100 \mu \mathrm{m}$ \\
\hline LS 3099 & $13266-5551$ & B1Iae & 10.8 & $62.4 \pm 3.4$ & 2008 May 21 & 0.76 & 35.9 & 35.43 & 11.66 \\
\hline LS 3268 & $14331-6535$ & B3Iab:e & 11.5 & $-29.8 \pm 2.1$ & 2008 May 20 & 4.40 & 108.7 & 70.71 & 20.61 \\
\hline LSE 3 & $17074-1845$ & B3IIIe & 11.5 & $12.4 \pm 1.5$ & 2008 May 22 & 0.5 & 12.20 & 5.66 & 3.47 \\
\hline TYC 6234-178-1 & $17203-1534$ & B1IIIpe & 12.5 & $51.4 \pm 2.1$ & 2008 May 23 & 0.32 & 10.7 & 6.88 & 3.37 \\
\hline LSE 76 & $17311-4924$ & B1IIe & 10.7 & $42.3 \pm 3.2$ & 2008 May 23 & 18.34 & 150.7 & 58.74 & 17.78 \\
\hline SAO 209306 & $17460-3114$ & O8III & 7.9 & - & 2008 May 22 & 6.26 & 20.82 & 18.6 & 220.4 \\
\hline LSS 4634 & $18023-3409$ & B2IIIe & 11.6 & $-3.4 \pm 3.8$ & 2008 May 20 & 0.26 & 2.94 & 1.82 & 25.64 \\
\hline LSE 63 & $18371-3159$ & B1Iabe & 11.9 & $10.7 \pm 1.0$ & 2008 May 21 & $0.25:$ & 6.31 & 5.16 & 1.95 \\
\hline LSE 148 & - & B6Ib & 10.2 & $-133.6 \pm 2.1$ & 2008 May 24 & - & - & - & - \\
\hline LS & 49 & B1Ibe & 11.4 & 1.7 & 2007 Sep. 04 & 0.29 & 10.26 & 6.45 & 1.77 \\
\hline
\end{tabular}

Figure 3 illustrates the spectra of our sample stars in the region around $\mathrm{H}_{\alpha}$, showing examples of emission profiles.

\subsubsection{SAO 209306 (IRAS 17460-3114)}

One object of our sample, SAO 209306, presents doubled He I lines in its spectrum, which can be interpreted as a spectroscopic evidence of binarity. This target was selected because its far-infrared (IRAS) fluxes are similar to post-AGB/PNe and because of its uncertain classification in the literature. SAO 209306 was described as a B3 star associated to an emission nebula by Sharpless (1953). Crampton (1971) classified it as a O7.5 star associated to the HII region Sharpless 15 based on UBV photometry. Ratag et al. (1990) and Umana et al. (2004) detected nebular radio emission typical of PNe using the VLA telescopes. Based on IRAS fluxes and spectral type, Parthasarathy et al. (2000) classified it as O8III hot post-AGB star. On the other hand, de Wit et al. (2005) described SAO 209306 as likely a field star associated to the open cluster Col347. Suarez et al. (2006), based on low-resolution spectra, classified it as a massive young object. Additionally, Crampton (1972) detected a variation of $213 \mathrm{~km} \mathrm{~s}^{-1}$ in the measured radial velocities and therefore they suspected variability or binarity for this object. The same argument was presented in the compilation of galactic O-type stars of Gies (1987), where this object appears as a suspected SB1 star, with spectral type $\mathrm{O} 8 \mathrm{~V}$.

In our spectrum we observe direct evidence that SAO 209306 is a double-lined spectroscopic binary (SB2) as shown in Fig. 4. 

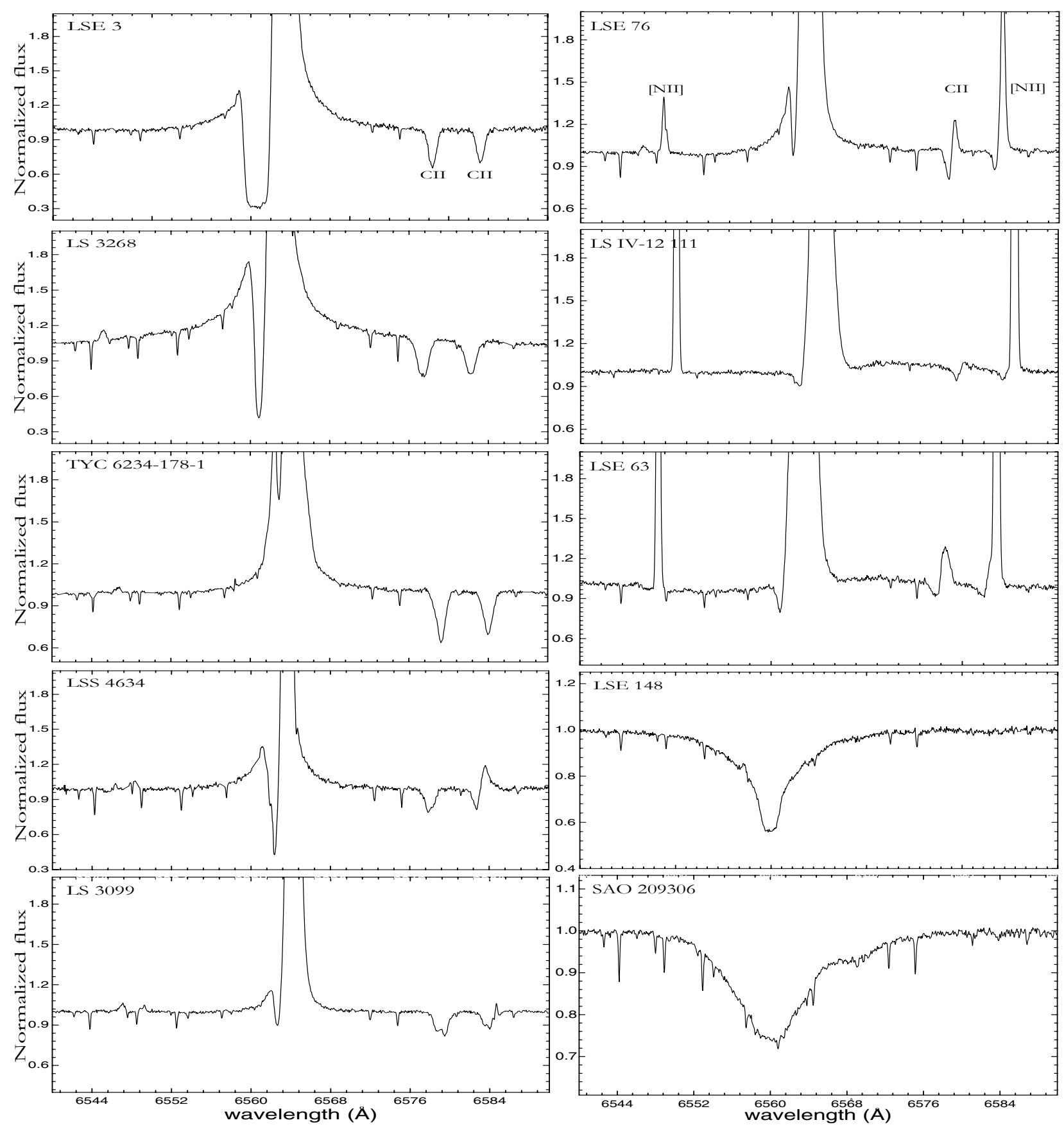

Fig. 3. Example spectra of the sample stars in the region around $\mathrm{H}_{\alpha}$ that may appear in absorption, strong emission, or as P-Cyg profile. Nebular lines of [N $\mathrm{NI}$ ] at $6548 \AA$ and $6584 \AA$ are also present in some spectra.

The high rotational projected velocity $(v \sin i)$ of this object does not allow us to clearly observe the metallic lines. However, we measured the radial velocities for the two stellar components for several helium lines and for a few metallic lines. No evidence of duplicity appears in the He Ir lines, suggesting that the secondary has slightly lower effective temperature $T_{\text {eff }}$ than the primary. Given the evidence of duplicity, we did not proceed with the chemical analysis for the star SAO 209306.

\subsection{Non-LTE model atmospheres}

The atmospheres of the hot post-AGB stars were analysed using the grids BSTAR2006 and OSTAR2002 model atmospheres generated with the code TLUSTY (Hubeny \& Lanz 1995). This code computes plane-parallel, horizontally homogeneous model atmospheres in radiative and hydrostatic equilibrium and provides a fully consistent non-LTE line blanketing.

The BSTAR2006 grid (Lanz \& Hubeny 2007) was calculated for 16 values of effective temperatures $\left(15000 \mathrm{~K} \leq T_{\mathrm{eff}} \leq\right.$ $30000 \mathrm{~K})$ with a step of $1000 \mathrm{~K}$ and for 13 values of surface gravity $(1.75 \leq \log g \leq 4.75)$ with a step of 0.25 dex. The microturbulent velocity is kept constant equal to $2 \mathrm{~km} \mathrm{~s}^{-1}$. The models were computed assuming six values of metallicity ranging from twice to one-tenth of the solar metallicity and metal-free. Additional model atmospheres for B supergiants $(\log g \leq 3.0)$ were calculated for a higher microturbulent velocity $\left(10 \mathrm{~km} \mathrm{~s}^{-1}\right)$ and a surface composition enriched in helium and nitrogen and 


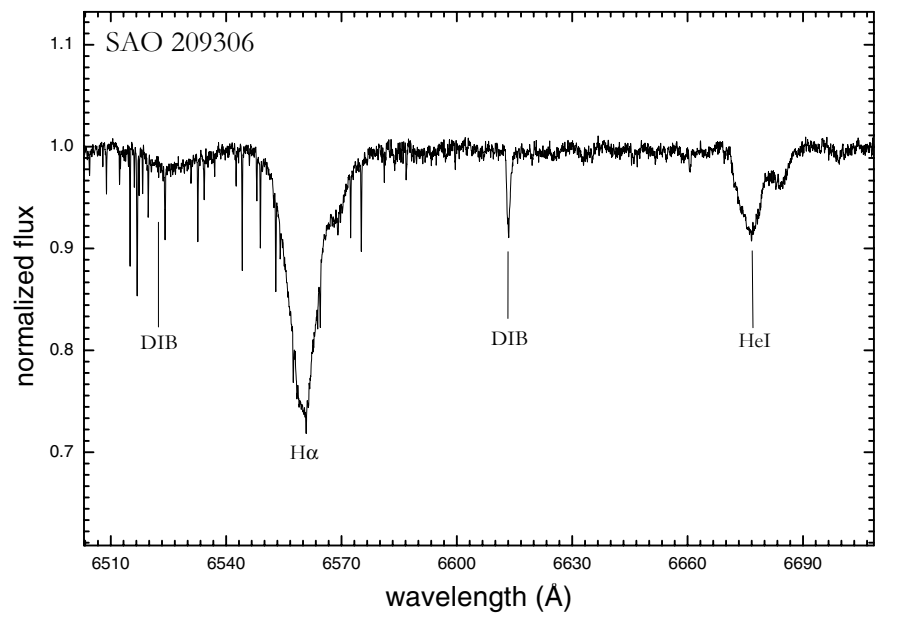

Fig. 4. Example of normalized spectrum of SAO 209306 (IRAS 174603114) showing the double profiles of $\mathrm{H}_{\alpha}$ and Her $6678 \AA$.

depleted in carbon. The OSTAR2002 grid (Lanz \& Hubeny 2003) was calculated for 12 values of effective temperatures $\left(27500 \mathrm{~K} \leq T_{\text {eff }} \leq 55000 \mathrm{~K}\right)$ with a step of $2500 \mathrm{~K}$ and for eight values of surface gravity $(3.0 \leq \log g \leq 4.75)$ with a step of $0.25 \mathrm{dex}$, and ten values of chemical composition, from metal-rich relative to the Sun to metal-free. In both grids, the lower limit of $\log g$ for a given effective temperature is set by an approximate location of the Eddington limit. The adopted chemical compositions were chosen to cover several typical environments of massive stars: the Galactic center, the Magellanic Clouds, blue compact dwarf galaxies like I $\mathrm{Zw} 18$, and galaxies at high redshifts.

The IDL interface Synplot ${ }^{2}$ was used to compute the emerging spectrum and to simulate the effects of rotational and instrumental broadening by means of convolution. Synplot calculates a synthetic spectrum for any $T_{\text {eff }}$ and $\log g$ (within the limits of the BSTAR2006 and OSTAR2002 grids) by interpolating the model structure, including non-LTE level populations, to desired $T_{\text {eff }}$ and $\log g$, and then computing a spectrum for such an interpolated model. This procedure was found more accurate than interpolating in the grid of synthetic spectra (Lanz \& Hubeny 2003).

\subsection{Physical properties of the stars: the atmospheric parameters}

In this section we describe the methods used to determine the stellar atmospheric parameters, effective temperaure $\left(T_{\text {eff }}\right)$, surface gravity $(\log g)$ and microturbulent velocity $(\xi)$ for the nine hot post-AGB stars.

\subsubsection{Effective temperature and surface gravity}

The wings of the emission-free profiles of Balmer lines were used to determine the first guess of surface gravity for a given $T_{\text {eff }}$ value. At the beggining of the Balmer series, the $\mathrm{H}$ profiles usually present strong emission in their cores, therefore, the Balmer lines H $73835 \AA$, H $63889 \AA$, and $\mathrm{H}_{\epsilon} 3970 \AA$ were chosen to derive $\log g$. Although P Cygni lines are present in several stars, which indicates the presence of the wind, we

\footnotetext{
${ }^{2}$ http://aegis.as.arizona.ed/ hubeny/pub/synplot.tar.gz
}

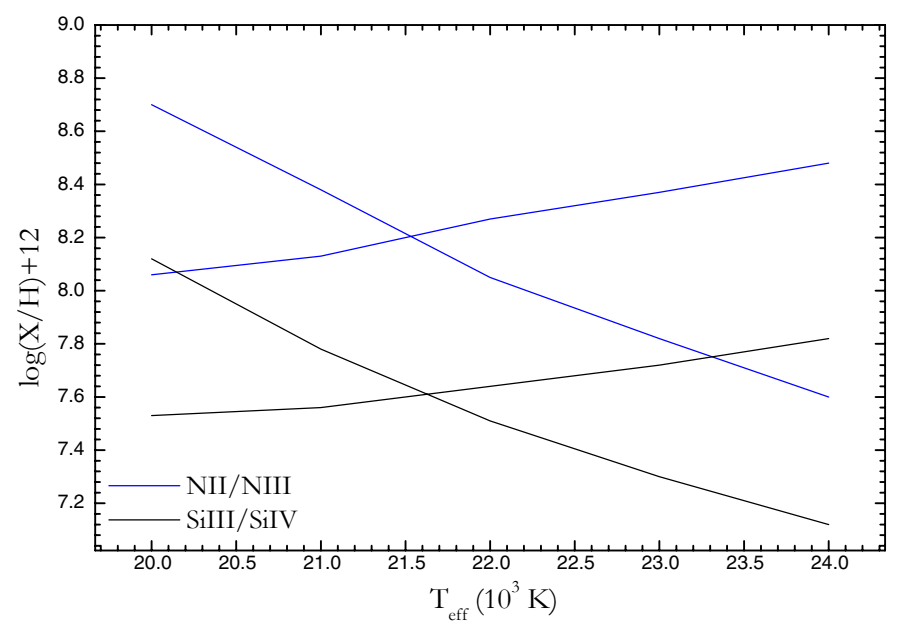

Fig. 5. Si III/IV and N II/III ionization balance for the hot post-AGB star LSE 63 (IRAS 18371-3159). The figure shows the average abundance results used to determine $T_{\text {eff }}=21600 \mathrm{~K}$ for this star.

did not consider stellar winds in our procedure. As discussed by Lanz \& Hubeny $(2003,2007)$, in all early type stars except in extreme supergiants and Wolf-Rayet stars, most of the spectral features used for deriving basic stellar parameters and chemical abundances are formed in the photosphere where velocities are low and geometrical extension is negligible. In our analysis, we excluded all emission lines and all lines that show asymmetries. Additionaly, the interestellar Ca II line at $3968 \AA$ was not accounted for in the models and we relied on the red wing of the $\mathrm{H}_{\epsilon}$ line. For some stars that presented weaker emission profiles, the models give a good fit even for $\mathrm{H}_{\delta}$, discarding the line cores. The higher Balmer lines were not used owing to a large uncertainty in the placement of the continuum and to a lower $\mathrm{S} / \mathrm{N}$ in the corresponding spectral region.

The adopted method is as follows: for a given object, we set a range of $T_{\text {eff }}$ value according to the stellar spectral type. The $\log g$ was allowed to vary to obtain the best fit to the observed Balmer lines for a given $T_{\text {eff }}$. The best fit was chosen by visual inspection and the $\mathrm{S} / \mathrm{N}$ was only considered qualitatively. We used high-quality spectra, with $S / N$ about $50-60$ in the region around $\mathrm{H} 7$, in the worst case. We repeated the process for the available $\mathrm{H}$ lines, and the surface gravities obtained for different lines were usually consistent within $0.1 \mathrm{dex}$. As a result we obtained five pairs ( $T_{\text {eff }}, \log g$, for which we were able to fit the $\mathrm{H}$ line profiles.

We used the pairs ( $T_{\text {eff }}, \log g$ ) as a starting point to derive the stellar $T_{\text {eff }}$ based on ionization balance. For most of the stars in our sample, we relied on the silicon ionization balance ( $\mathrm{Si} \mathrm{III/IV)}$ ). The ionization balance for other species such as $\mathrm{N}$ II/ $/ \mathrm{N}_{\text {III }}$ and O II/O III were used to check the derived $T_{\text {eff }}$ for three objects and the results agreed perfectly with the values of $T_{\text {eff }}$ obtained from the silicon balance. The sulfur balance ( $\left.\mathrm{S}_{\text {II }} / \mathrm{III}\right)$ was used to define $T_{\text {eff }}$ for the two coolest objects. The ionization balance of helium $(\mathrm{He} \mathrm{I} / \mathrm{II})$ was considered in the analysis of the LSE 148.

In Fig. 5 we show the $T_{\text {eff }}$ determination using Si III/IV and $\mathrm{N}$ II/III ionization balance for the star LSE 63. Once $T_{\text {eff }}$ was constrained, new fits of the Balmer lines wings were performed and the surface gravity was finally determined for the object.

We estimated the uncertainty in the $T_{\text {eff }}$ based on the abundance dispersion obtained from the analysis of the ionization balance. The difference between the mean abundance derived 
Table 2. The atmospheric parameters and $v \sin i$ derived for hot post-AGB stars.

\begin{tabular}{lccccc}
\hline \hline $\begin{array}{l}\text { Object } \\
\text { (Name) }\end{array}$ & $\begin{array}{c}T_{\text {eff }} \\
(\mathrm{K})\end{array}$ & Method & $\begin{array}{c}\log g \\
(\mathrm{cgs})\end{array}$ & $\begin{array}{c}\xi \\
\left(\mathrm{km} \mathrm{s}^{-1}\right)\end{array}$ & $\begin{array}{c}v \sin i \\
\left(\mathrm{~km} \mathrm{~s}^{-1}\right)\end{array}$ \\
\hline LS 3099 & $20200_{-400}^{+300}$ & Si III/IV & 2.38 & 16 & $39 \pm 3$ \\
LS 3268 & $16200_{-200}^{+300}$ & $\mathrm{~S}$ II/III & 2.04 & 17 & $38 \pm 5$ \\
LSE 3 & $15300_{-600}^{+600}$ & $\mathrm{~S}_{\text {II } / \mathrm{III}}$ & 2.05 & 10 & $31 \pm 3$ \\
TYC 6234-178-1 & $19000_{-500}^{+200}$ & $\mathrm{Si} \mathrm{III/IV}$ & 2.25 & 14 & $35 \pm 2$ \\
LSE 76 & $20500_{-500}^{+500}$ & Si III/IV & 2.35 & 16 & $26 \pm 4$ \\
LSS 4634 & $19400_{-400}^{+300}$ & Si III/IV & 2.28 & 12 & $35 \pm 3$ \\
LSE 63 & $21600_{-700}^{+700}$ & Si III/IV & 2.58 & 20 & $46 \pm 4$ \\
LSE 148 & $30900_{-300}^{+300}$ & He I/II & 3.83 & 3 & $2 \pm 2$ \\
LS IV-12 111 & $21000_{-500}^{+600}$ & Si III/IV & 2.42 & 20 & $33 \pm 4$ \\
\hline
\end{tabular}

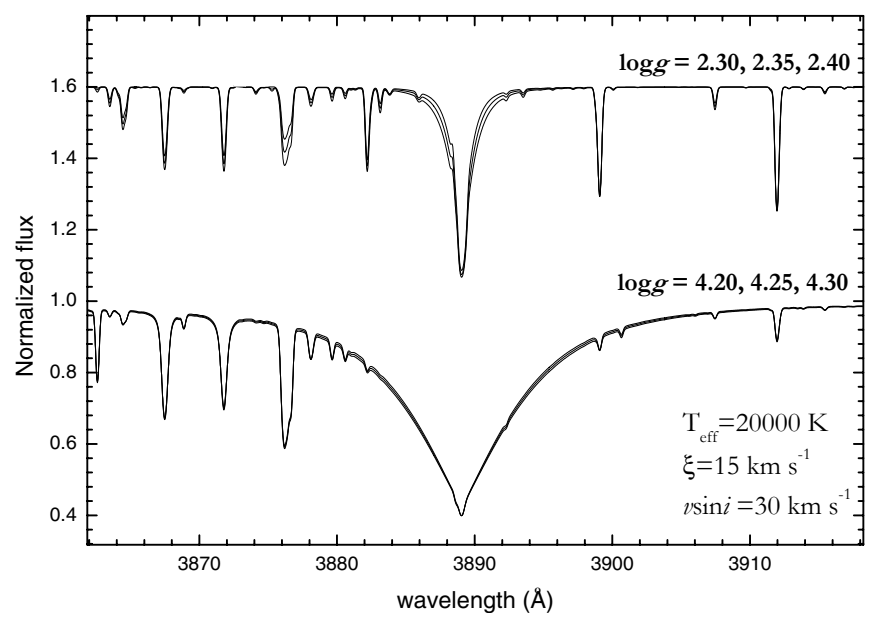

Fig. 6. Comparison between wings of hydrogren profiles computed for dwarf (bottom) and evolved stars (top), considering different values of $\log g$, in steps of 0.05 dex. The lower the value of $\log g$, the higher the sensivity of the line wings to the surface gravity.

for a given element in two consecutive ionization stages, for example, Si III and Si Iv, will not exceed the abundance dispersion obtained from the individual lines. This procedure allows one to estimate the $T_{\text {eff }}$ range in which the equilibrium temperature is reached. The $T_{\text {eff }}$ uncertainties may vary according to the analysis of different species.

We estimated the uncertainty on the surface gravity from several fits of hydrogen Balmer lines for a given $T_{\text {eff }}$ and considering the minimum difference in $\log g$. The broadened wings of hydrogen lines of evolved stars are more sensitive to variations in $\log g$ than those of main sequence stars. This is illustrated in Fig. 6, where we present a set of theoretical profiles of H 6 at $3889 \AA$. In this figure, the profiles were computed for values of surface gravity that are typical for post-AGB stars and for main sequence stars. We note the different behaviour of the line wings according to the different values of gravity. Considering this result, we estimate the uncertainty of 0.05 dex for $\log g$.

\subsubsection{Microturbulent velocity}

The microturbulent velocity $\xi$ for all objects were derived from $\mathrm{O}_{\text {II }}$ (and occasionally $\mathrm{N}_{\text {II }}$ ) lines requiring that the abundances derived from the individual lines are independent of the line strength (i.e., a slope close to zero is obtained in a plot of equivalent widths (EW) versus abundances). Figure 7 shows

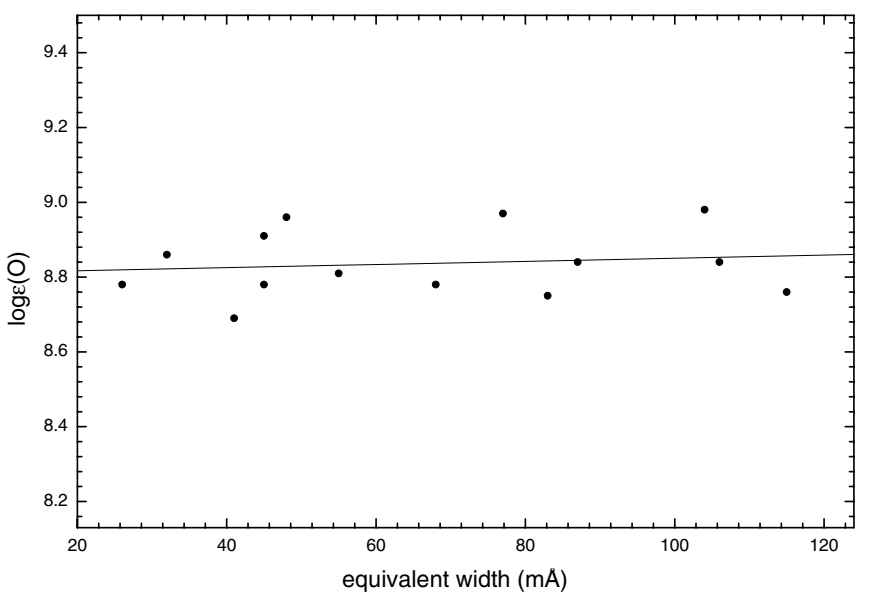

Fig. 7. Microturbulent velocity diagnostic for the hot post-AGB star LSE 76 using the equivalent width versus abundances diagram of $\mathrm{O}$ II lines. The diagram also shows the linear fit to the measured $\mathrm{O}_{\text {II }}$ lines with a slope close do zero, indicating the best value of $\xi$.

an example of the microturbulent velocity analysis for the star LSE 76. Our results indicate high values of microturbulent velocity, as can be see in the Table 2. Microturbulent velocities of evolved massive OB stars typically exceed $10 \mathrm{~km} \mathrm{~s}^{-1}$, as reported in many papers (Gies \& Lambert 1992; to Cantiello et al. 2009). The LTE approach produces even higher (supersonic) microturbulent velocity values. Self-consistent non-LTE analysis leads to significantly lower $\xi$, but significant $\xi$ values are usually needed to properly fit the line profiles of supergiants. The uncertainty of the microturbulent velocity was estimated by chosing a region of minimal dispersion in the plane $E W \times \log \epsilon(\mathrm{O})$. A typical value of $\delta \xi=2 \mathrm{~km} \mathrm{~s}^{-1}$ was found for most of the objects, except for LSE 3 and LSS 4634. The $\xi$ value of the star LSE 3 was derived from relatively weaker $\mathrm{O}$ II lines and is slightly more uncertain. The quality of the observed spectrum of the star LSS 4634 affected the profiles of some $\mathrm{O}_{\mathrm{II}}$ lines and resulted in a larger uncertainty for the derived value.

\subsubsection{Rotational velocity}

The projected rotational velocity $v \sin i$ was considered as a free parameter in our analysis. As mentioned in Sect. 3.2, the Synplot interface allows us to convolve the synthetic spectrum with arbitrary values of $v \sin i$ to obtain a best fit (the instrumental profile was also considered as a broadening mechanism). The stellar 
D. R. C. Mello et al.: Chemical abundances of hot post-AGB stars

Table 3. Absolute abundances for the sample stars on a logarithmic scale with $\log \epsilon(\mathrm{H})=12$.

\begin{tabular}{lccccccc}
\hline \hline Object & $\log \epsilon(\mathrm{He})$ & $\log \epsilon(\mathrm{C})$ & $\log \epsilon(\mathrm{N})$ & $\log \epsilon(\mathrm{O})$ & $\log \epsilon(\mathrm{Mg})$ & $\log \epsilon(\mathrm{Si})$ & $\log \epsilon(\mathrm{S})$ \\
\hline LS 3099 & $11.10 \pm 0.08(9)$ & $8.27 \pm 0.12(9)$ & $8.50 \pm 0.08(13)$ & $8.85 \pm 0.14(14)$ & 7.78 & $7.78 \pm 0.10(6)$ & $6.93 \pm 0.09(5)$ \\
LS 3268 & $10.90 \pm 0.04(7)$ & $8.28 \pm 0.09(8)$ & $8.09 \pm 0.08(14)$ & $8.86 \pm 0.08(19)$ & - & $7.41 \pm 0.12(4)$ & $7.11 \pm 0.06(13)$ \\
LSE 3 & $10.88 \pm 0.05(8)$ & $8.14 \pm 0.19(6)$ & $7.85 \pm 0.08(10)$ & $8.74 \pm 0.07(9)$ & 7.67 & $7.34 \pm 0.06(4)$ & $6.84 \pm 0.16(10)$ \\
TYC 6234-178-1 & $11.14 \pm 0.06(11)$ & $8.23 \pm 0.08(8)$ & $8.00 \pm 0.15(15)$ & $8.71 \pm 0.10(18)$ & 7.56 & $7.31 \pm 0.06(5)$ & $6.64 \pm 0.07(3)$ \\
LSE 76 & $11.05 \pm 0.06(6)$ & $8.27 \pm 0.08(5)$ & $8.22 \pm 0.08(13)$ & $8.84 \pm 0.12(24)$ & 7.47 & $7.42 \pm 0.14(5)$ & $6.57 \pm 0.16(4)$ \\
LSS 4634 & $11.13 \pm 0.04(5)$ & $8.03 \pm 0.20(4)$ & $8.59 \pm 0.10(14)$ & $8.58 \pm 0.07(10)$ & - & $7.28 \pm 0.12(5)$ & $6.62 \pm 0.17(3)$ \\
LSE 63 & $10.93 \pm 0.05(3)$ & - & $8.13 \pm 0.14(8)$ & $8.76 \pm 0.20(15)$ & - & $7.60 \pm 0.22(6)$ & $6.81 \pm 0.06(2)$ \\
LSE 148 & $11.09 \pm 0.04(9)$ & - & 7.23 & $7.43 \pm 0.12(8)$ & 6.35 & $6.12 \pm 0.07(4)$ & - \\
LS IV-12 111 & $10.94 \pm 0.03(6)$ & $8.13 \pm 0.06(4)$ & $7.99 \pm 0.11(10)$ & $8.86 \pm 0.17(32)$ & 7.23 & $7.38 \pm 0.19(6)$ & $6.65 \pm 0.12(2)$ \\
Sun & 10.93 & 8.39 & 7.78 & 8.66 & 7.53 & 7.51 & 7.15 \\
\hline
\end{tabular}
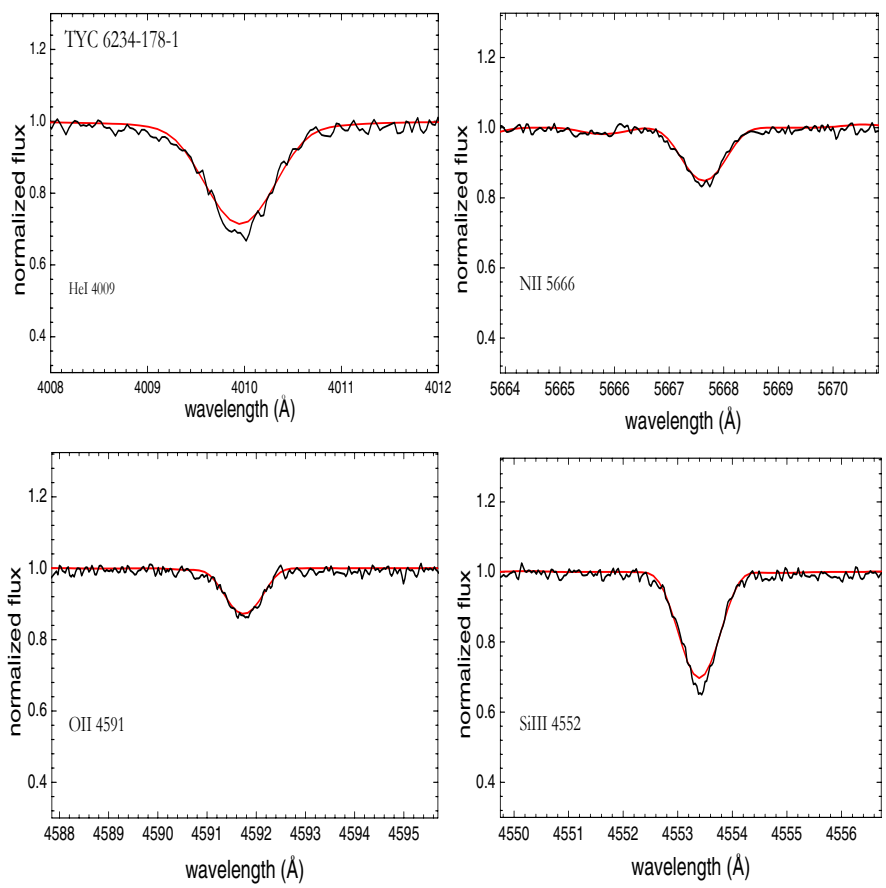

Fig. 8. Examples of line fits for the star TYC 6234-178-1 (IRAS 172031534). Observed profiles of lines He I4009, N II5666, O II 4591, and Si III $4552 \AA$ are superposed on non-LTE theoretical spectra (red lines).

$v \sin i$ was taken as the average obtained from individual line fits, with the corresponding standard deviation. In Table 2 we list the atmospheric parameters and projected rotational velocity for all objects of our sample.

\subsection{Chemical abundances}

We used the atmospheric parameters discussed in the previous sections to derive absolute abundances for 9 hot post-AGB stars. The non-LTE chemical abundances of $\mathrm{He}, \mathrm{C}, \mathrm{N}, \mathrm{O}, \mathrm{Mg}$, Si, and S were determined on line-by-line basis by comparing the observed spectra with theoretical profiles generated with the code Synplot. Table 3 lists the mean abundances for the objects with the respective standard deviation and the number of analysed lines indicated in parenthesis. The solar abundances are from Asplund et al. (2006). In Fig. 8 we show some line fits of the star TYC 6234-178-1 (IRAS 17203-1534).

Several He I lines are present in the spectra of the sample stars, and He II lines are also present in the spectra of the hottest objects. Most of the analysed hot post-AGB stars show He I lines in P-Cyg profiles, or in other cases, some He I lines have apparent signs of wind contamination. This is the case for the well-known diffuse He I lines at 4026, 4387, 4471, and $4921 \AA$ A. Some He I lines in the red region of our spectra also present a similar behaviour. We therefore took special care to select helium lines free of wind contamination. As listed in Table 3, the mean helium abundances for the all object is solar or slightly enriched. The ionization balance He I/II was used to determine the effective temperature for one object: LSE 148. In this case we use

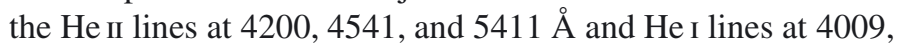
4026, 4387, 4921, 5015, and $6678 \AA$.

Carbon underabundance in hot post-AGB stars have been cited by several authors as an outstanding characteristic (Van Winckel 2003). They indicate that hot post-AGB stars may have left the AGB phase before undergoing the third dredge-up. Our results suggest underabundance of carbon with $\log \epsilon(\mathrm{C})$ values in the range 8.03-8.28. These results are generally higher than previous abundances presented in the literature for hot postAGB stars (McCausland et al. 1992; Moehler \& Heber 1998). However, we note that most of these previous carbon results are based on an LTE analysis of the $C_{\text {II }}$ line at $4267 \AA$, which is known to be very sensitive to non-LTE effects (Nieva \& Przybilla 2008; Trundle et al. 2007). The carbon abundances for our sample stars were obtained from the synthesis of $\mathrm{C}_{\text {II }}$ lines at $\sim 5120-5152 \AA$ and $\sim 6780-6800 \AA$, which are fully accounted for in the carbon model atom used by TLUSTY.

Several nitrogen lines, predominantly $\mathrm{N}_{\text {II }}$ lines, are present in our spectra. For the hotter objects, N III lines can be also detected. Low-excitation nebular lines of nitrogen are also present for some hot post-AGB stars, as shown in Fig. 3. We obtained a wide range of nitrogen abundances in our analysis and some of the analysed stars exhibit significant nitrogen enhancement. The mixture epsisode known as the second dredge-up accompanies the formation of an electron-degenerate $\mathrm{C}$-O core in the horizontal branch of intermediate- and low-mass stars. Depending on the progenitor mass, the convective envolope deepens downwards to the helium-hydrogen intershell, dredging CNO-processed material to the stellar atmosphere (Herwig 2005). The apparent nitrogen enrichment of our objects can be related to the second dredge-up event or the "hot bottom burning" (hereafter, HBB) process. More details are discussed in Sect. 4.3.3.

Strong O II lines are present in the spectra of our hot postAGB stars. Among the most intense lines, we cite the $\mathrm{O}$ II lines at 4075, 4591, 4596, 4541, and $4661 \AA$. The microturbulent velocity was constrained using $\mathrm{O}_{\text {II }}$ lines, since this specie presents very many spectral lines with a wide range of relative strength. We obtained solar or slightly higher oxygen abundances for our sample stars, except for the metal-poor star LSE 148, for which we obtained $\log \epsilon(\mathrm{O})=7.43$. 
Table 4. Abundance uncertainties.

\begin{tabular}{ccccccc}
\hline \hline Object & Species & $\delta\left(T_{\text {eff }}\right)$ & $\delta(\log g)$ & $\delta(\xi)$ & $\delta(v \sin i)$ & $\delta$ (total $)$ \\
\hline LSE 3 & $\mathrm{He}_{\text {I }}$ & -0.07 & -0.02 & -0.05 & +0.05 & 0.10 \\
& $\mathrm{C}_{\text {II }}$ & -0.08 & -0.02 & -0.03 & +0.05 & 0.10 \\
& $\mathrm{~N}_{\text {II }}$ & -0.10 & +0.03 & -0.04 & +0.06 & 0.12 \\
& $\mathrm{O}_{\text {II }}$ & -0.18 & +0.02 & -0.06 & +0.05 & 0.19 \\
& $\mathrm{Mg}_{\text {II }}$ & +0.17 & -0.03 & -0.04 & +0.05 & 0.18 \\
& $\mathrm{Si}_{\text {III }}$ & -0.14 & +0.02 & -0.05 & +0.06 & 0.16 \\
& $\mathrm{~S}_{\text {III }}$ & +0.09 & -0.01 & -0.02 & +0.05 & 0.11 \\
\hline \multirow{2}{*}{ LSE 63 } & $\mathrm{He}_{\text {I }}$ & +0.04 & -0.05 & -0.02 & +0.02 & 0.07 \\
& $\mathrm{~N}_{\text {II }}$ & +0.11 & -0.06 & -0.02 & +0.03 & 0.13 \\
& $\mathrm{O}_{\text {II }}$ & +0.05 & -0.03 & -0.03 & +0.05 & 0.09 \\
& $\mathrm{Si}_{\text {III }}$ & -0.06 & -0.03 & -0.05 & +0.07 & 0.10 \\
& $\mathrm{~S}_{\text {III }}$ & +0.01 & -0.02 & -0.01 & +0.04 & 0.05 \\
\hline LSE 148 & $\mathrm{He}_{\text {I }}$ & +0.05 & +0.02 & -0.01 & +0.02 & 0.06 \\
& $\mathrm{~N}_{\text {III }}$ & -0.07 & +0.06 & -0.03 & +0.04 & 0.11 \\
& $\mathrm{O}_{\text {II }}$ & +0.08 & -0.06 & -0.03 & +0.03 & 0.11 \\
& $\mathrm{Mg}_{\text {II }}$ & +0.05 & +0.03 & -0.05 & +0.03 & 0.08 \\
& $\mathrm{Si}_{\text {III }}$ & +0.10 & -0.05 & -0.03 & +0.04 & 0.12 \\
\hline
\end{tabular}

The analysis of magnesium was based on the only $\mathrm{Mg}$ II line visible in the observed spectral range, at $4481 \AA$. The derived magnesium abundances are scattered around the solar value, ranging from $[\mathrm{Mg} / \mathrm{H}]=+0.25$ dex for LS 3099 to $-0.3 \mathrm{dex}$ for LS IV-12 111. The asymmetrical profiles of the Mg II prevent the determination of magnesium abundance for the objects LSS 4634, LSE 63, and LS 3268.

Absorption lines of Si II, Si III, and Si IV can be identified in the spectra of our sample stars. Most of the observed spectra present strong lines of $\mathrm{Si}$ II and $\mathrm{Si}$ III and, for the hotter objects, Si Iv lines at 4116 and $4654 \AA$ are also present. Although many $\mathrm{Si}$ II lines were detected in the observed spectra, Si II was not used to derive silicon abundances or to constrain the stellar parameters, since most of $\mathrm{Si}$ II lines usually appear in emission. We mainly used the Si III/IV ionization balance to estimate the effective temperature. The lines of the silicon triplet at 4552 , 4567, and $4574 \AA$ are the most prominent Si III profiles, while the line at $4116 \AA$ is the more important Si IV profile used to define $T_{\text {eff }}$. The silicon abundances listed on the Table 3 span from 7.28 to 7.78, excluding the metal-poor star LSE 148.

Like magnesium and silicon, sulfur is also an $\alpha$-element. In our spectra we detected several absorption lines of $\mathrm{S}_{\text {II }}$ and $\mathrm{S}$ III and the sulfur ionization balance was used to derive $T_{\text {eff }}$ for the coldest objects LSE 3 and LS 3268. Forbbiden and permitted sulfur emission lines are also present for some objects, especially for the hotter stars. All obtained sulphur abudances are sub-solar.

\subsubsection{Abundance uncertainties}

Table 3 shows the mean abundances obtained with the respective standard deviations. We analysed the abundance uncertainties caused by the atmospheric parameters (effective temperature, surface gravity, and microturbulent velocity), the rotational velocity $v \sin i$ and the placement of the continuum $(\delta$ continuum $=$ 0.01 for all stars) for the stars LSE 3, LSE 63, and LSE 148. These stars were chosen to cover the effective temperature interval of our sample. We varied the values of the stellar parameters by adding the corresponding uncertainty, one at a time, keeping the other parameters constant, and re-derived the abundances. The abundance variations obtained for the different parameters are listed in Table 4. In the last column of this table we list the total uncertainties obtained from the sum of the uncertainties in quadrature.

\section{Discussion}

\subsection{Masses and metallicity}

Our abundance results combined with other published results (infrared colours similar to PNe, mass loss occurring for some objects, circumstelar envelope evidenced by emission lines) indicate that the objects studied in this paper are indeed in their post-AGB phase, except, possibly, LSE 148. Using the $\log T_{\text {eff }}-$ $\log g$ diagram, we estimate the masses of our targets using the evolutionary tracks of Schönberner (1983) for post-AGB stars for core masses of 0.546 and $0.565 M_{\odot}$ and evolutionary tracks of Blöcker (1995) with core masses of $0.605,0.625,0.696$, 0.836 and $0.940 M_{\odot}$, as can be seen in the Fig. 9. In this figure we also indicate the post-HB evolutinary tracks from Dorman et al. (1993) computed for core masses of 0.49, 0.5, 0.51 and $0.53 M_{\odot}$ and some sdOB stars from Heber (1986) and Ahmad \& Jeffery (2003) for comparison. We estimate the core mass $M_{\mathrm{c}} \sim 0.63 M_{\odot}$ for LSE 3 and $M_{\mathrm{c}} \sim 0.70 M_{\odot}$ for LS 3268, LSE 63 and LS 3099. For the objects LS IV-12 111, LSE 76, LSS 4634 and TYC 6234-178-1, we estimate $M_{\mathrm{c}} \sim 0.84 M_{\odot}$ and for the hottest object LSE 148 we estimate a core mass $M_{\mathrm{c}} \sim 0.55 M_{\odot}$. Based on this analysis, LS IV-12 111, LSE 76, LSS 4634, TYC 6234-178-1, LS 3099, LSE 63, and LS 3268 can be considered to be massive post-AGB stars, indicating a rapid evolution to the PNe phase. In this case, it is expected that the more massive objects evolve rapidly from the AGB to planetary nebulae in $10^{2}-10^{3}$ years (Schönberner 1979a,b). Some hot post-AGB stars have been monitored in the last decades and some objects have shown considerable photometric and spectroscopic variations, e.g. the objects V1853 Cyg and HD 341617 (Arkhipova et al. 2002). On the other hand, LSE 3 is likely a less massive object, evolving to PNe phase more slowly. LSE 148 is situated among the low-mass stars with a long evolutionary time to reach the locus of the PNe. Using the initial-to-final mass relation $M_{\mathrm{i}} / M_{\mathrm{f}}$ (Weidemann 1987, 2000), we can estimate the zero-age main sequence (ZAMS) masses of our objects. Thus, it seems that the more massive objects (seven of them) have an initial mass of $M_{\mathrm{i}} \geq 4 M_{\odot}$, while the less massive (LSE 3 and LSE 148) have $M_{\mathrm{i}} \leq 3.0 M_{\odot}$. However, the mass estimates are influenced by several relevant parameters, including the uncertainties due to the atmospheric parameters, especially the surface gravity and the adopted models in the computation of the tracks. Considering the uncertainty of $0.05 \mathrm{dex}$ in $\log g$ will impart an 
D. R. C. Mello et al.: Chemical abundances of hot post-AGB stars

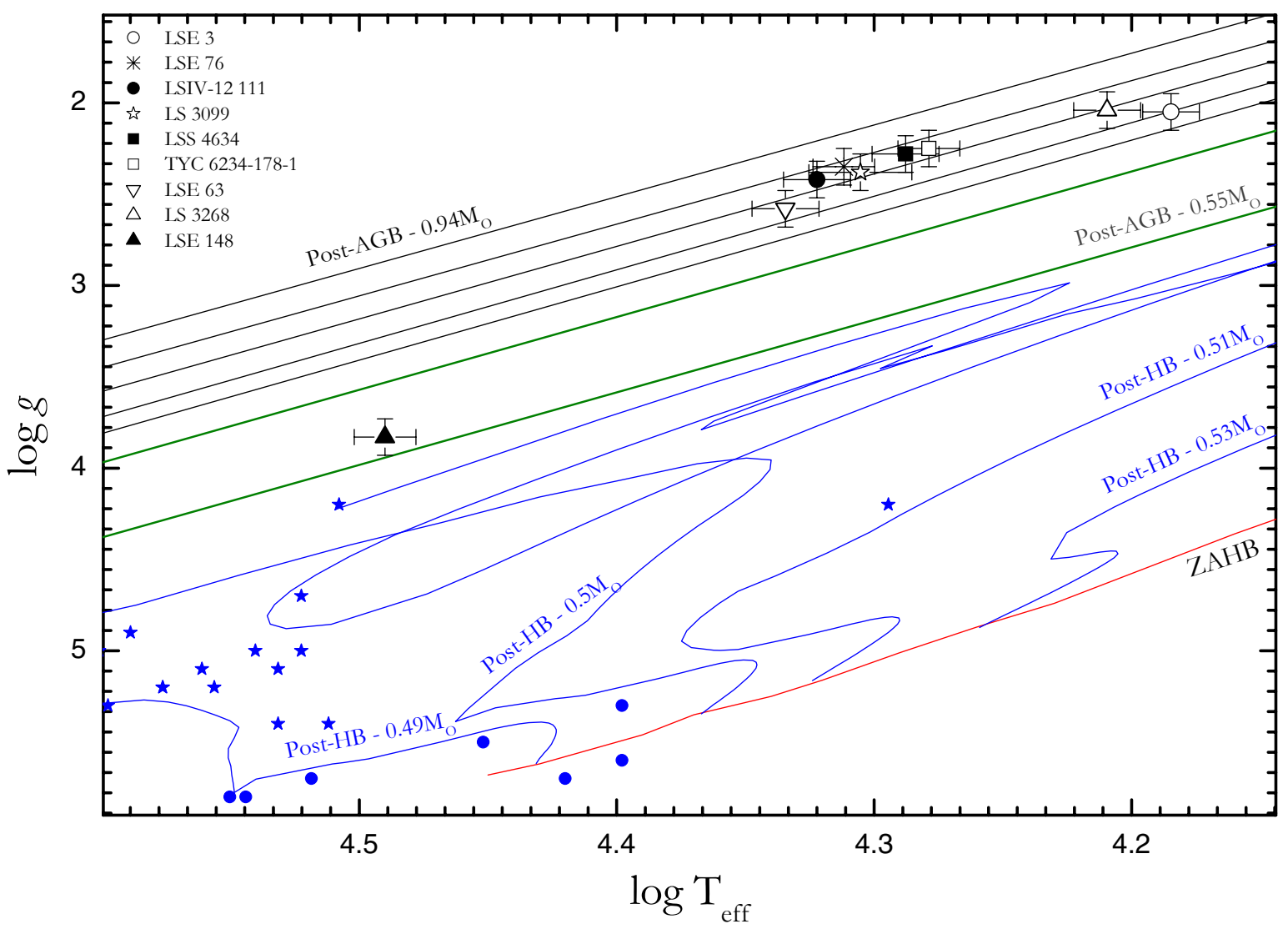

Fig. 9. Positions of the hot post-AGB stars in the $\log T_{\text {eff }}-\log g$ diagram. The blue lines denote the post-HB evolutionary tracks according to Dorman et al. (1993) with core mass of $0.49,0.5,0.51$ and $0.53 M_{\odot}$ and the red line denote the zero-age horizontal branch (ZAHB). The black lines denote the post-AGB evolutionary tracks according to Blöcker (1995) for core stellar masses of 0.605, 0625, 0.696, 0.836, and 0.940 $M_{\odot}$ and the green lines represent the post-AGB evolutionary tracks according to Schönberner (1983) for core stellar masses of 0.546 and $0.565 M_{\odot}$. For comparison, we include some sdOB stars (blue stars and filled blue circles) of the samples of Heber (1986) and Ahmad \& Jeffery (2003).

Table 5. Core mass, ZAMS mass and metallicity estimates for the objects.

\begin{tabular}{lccc}
\hline \hline $\begin{array}{l}\text { Object } \\
\text { (Name) }\end{array}$ & $\begin{array}{c}M_{\mathrm{c}} \\
\left(M_{\odot}\right)\end{array}$ & $\begin{array}{c}M_{\text {ZAMS }} \\
\left(M_{\odot}\right)\end{array}$ & $Z(\mathrm{CNO})$ \\
\hline LS 3099 & 0.70 & 4.0 & 0.018 \\
LS 3268 & 0.70 & 4.0 & 0.016 \\
LSE 3 & 0.63 & 3.0 & 0.012 \\
TYC 6234-178-1 & 0.84 & 5.0 & 0.012 \\
LSE 76 & 0.84 & 5.0 & 0.016 \\
LSS 4634 & 0.84 & 5.0 & 0.013 \\
LSE 63 & 0.70 & 4.0 & 0.011 \\
LSE 148 & 0.55 & 1.0 & 0.001 \\
LS IV-12 111 & 0.84 & 5.0 & 0.015 \\
\hline
\end{tabular}

uncertainty of $\sim 0.20 M_{\odot}$ in the estimated core mass, which may represent an uncertainty of $2.0 M_{\odot}$ for the estimated ZAMS mass $\left(M_{\text {ZAMS }}\right)$ of the object. Estimates of the core mass $\left(M_{\mathrm{c}}\right)$ and ZAMS mass are listed in Table 5.

Iron lines are very weak in the spectra of early-type stars, making the estimate of metallicity based on iron a very difficult task. For the hot post-AGB stars of our sample, this problem is even worse since most of the iron lines are in emission. Therefore we must rely on the $\mathrm{CNO}$ abundances as metallicity proxies. The results of $Z(\mathrm{CNO})$ are also shown in Table 5 and indicate approximately solar metallicity for all objects of the sample, except for LSE 148.

\subsection{Notes on individual objects}

\section{LSE 3 (IRAS 17074-1845)}

This source was firstly described as an early-type emissionline star by Henize (1976). It appears in the Thé catalogue (Thé et al. 1994) as a Be star with an associated nebula. Garcia-Lário et al. (1997) obtained near-IR observations for this star and classified it as a post-AGB star. In a study based on lowresolution spectra, Parthasarathy et al. (2000) classified it as a hot post-AGB B3IIIe star based on its high galactic latitude, farinfrared colours similar to $\mathrm{PNe}$, and Be spectral type. Using lowresolution UV(IUE) spectra, Gauba \& Parthasarathy (2003) estimated the effective temperature and surface gravity as being $17000 \mathrm{~K}$ and 3.4 dex, respectively. In their search for young PNe, Umana et al. (2004) did not detect signs of nebular radio emission for IRAS 17074-1845, indicating that the surrounding nebula was not ionized by the central star yet. Suarez et al. (2006) classified LSE 3 as a transition object based on its optical low-resolution spectra.

Our spectrum of LSE 3 shows P-Cyg profiles at $\mathrm{H}_{\gamma}, \mathrm{H}_{\beta}$, and $\mathrm{H}_{\alpha}$ with a broad blueshifted absorption. Some $\mathrm{He}_{\mathrm{I}}$ lines also show emission components. Additionally, several forbbiden emission lines of Fe II can be detected for this source. The obtained parameters for this object $\left(T_{\text {eff }}=15300 \mathrm{~K}, \log g=2.05\right)$ are consistent with the effective temperature estimated by Gauba \& Parthasarathy (2003) based on low-resolution UV spectra. However, our result for surface gravity is 1.4 dex lower than their 


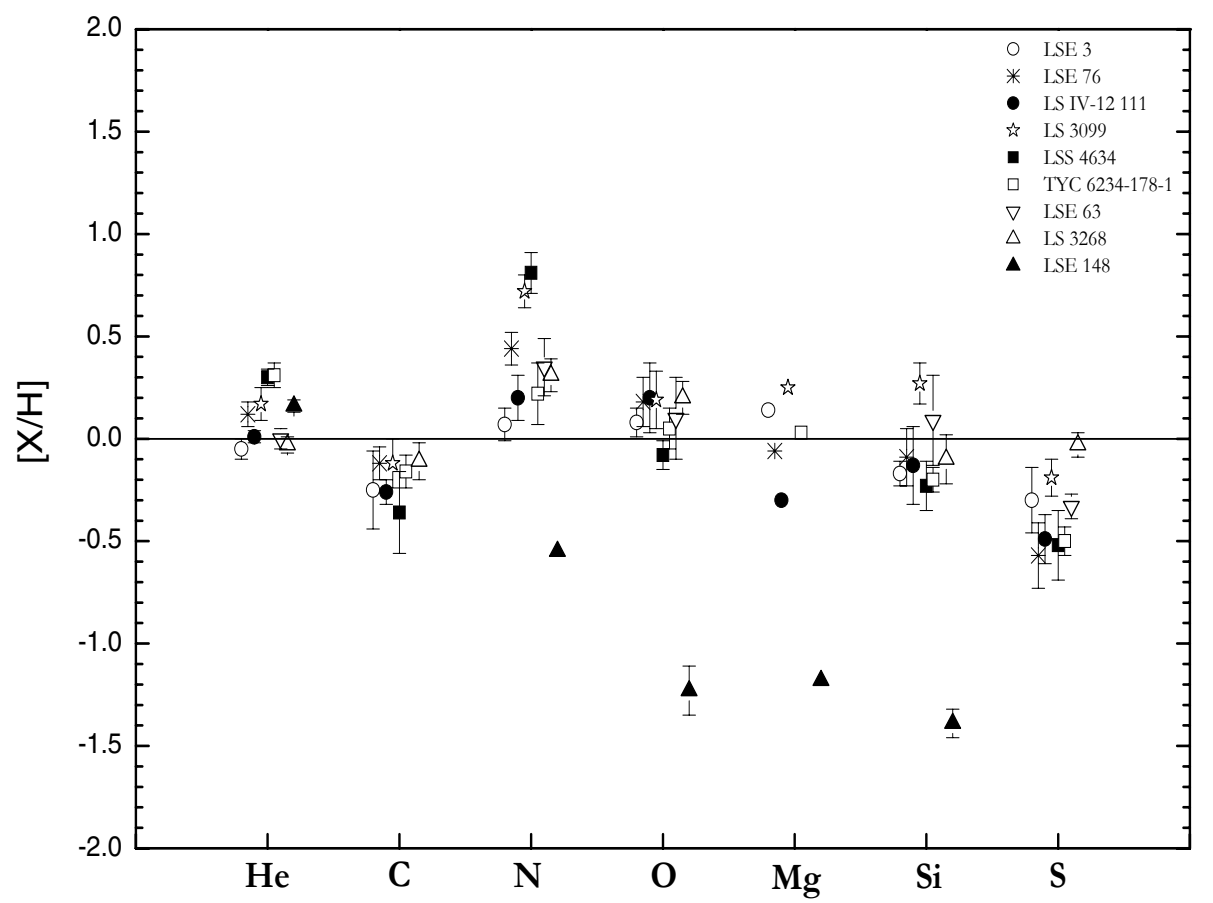

Fig. 10. Relative chemical abundances derived for our sample objects, represented by different symbols. The black line shows the solar abundances reference by Asplund et al. (2006).

value. Gauba \& Parthasarathy (2003) pointed out that the uncertainty in the determination of the surface gravity, based on the spectral classification, may be \pm 1.0 dex.

\section{LS 3268 (IRAS 14331-6435)}

Henize (1976) detected emission in $\mathrm{H}_{\alpha}$ for this source. Kozok (1985) studied the distribution of Be stars in the Galactic central region and derived a distance to the Sun of $1.9 \mathrm{kpc}$ and $M_{V}=-3.08$ for this object. Parthasarathy et al. (2000), based on low-resolution spectra, classified it as B3Ie hot post-AGB star based on the detected emission in $\mathrm{H}_{\beta}$. The post-AGB nature was later confirmed by Suarez et al. (2006), using low-resolution spectra.

The observed spectrum of LS 3268 is very similar to that of LSE 3. The Balmer lines $\mathrm{H}_{\gamma}$ and $\mathrm{H}_{\beta}$ are in P-Cyg and $\mathrm{H}_{\alpha}$ in emission. Gauba \& Parthasarathy (2003) have estimated the atmospheric parameters for this object based on spectral type and obtained $T_{\text {eff }}=16200 \mathrm{~K}$ and $\log g=2.6 \mathrm{dex}$. In our analysis, we obtained $T_{\text {eff }}=16200 \mathrm{~K}$ (in perfect agreement) and our estimated $\log g$ is 0.56 dex lower. The analysis of magnesium was not possible for this star, since the $\mathrm{Mg}$ II line at $4481 \AA$ shows a highly asymmetrical profile.

\section{TYC 6234-178-1 (IRAS 17203-1534)}

This source was classified by Parthasarathy et al. (2000) as a B1IIIpe hot post-AGB star, based on high galactic latitude, IRAS colours similar to PNe, and low-resolution spectra. Gauba \& Parthasarathy (2003) derived effective temperature and surface gravity for this target by modelling the UV(IUE) lowresolution spectra using LTE model atmospheres (Kurucz 1993) with solar metallicity, considering the circumstellar extinction for IRAS 17203-1534 as negligible and obtained $T_{\text {eff }}=19000 \mathrm{~K}$ and $\log g=2.5$ dex. In their paper, Suarez et al. (2006) classified this source as a post-AGB star. Umana et al. (2004) have detected no nebular radio emission surrounding the central star in their search for very young PNe.

Our observed spectrum presents the Balmer lines $\mathrm{H}_{\gamma}, \mathrm{H}_{\beta}$ and $\mathrm{H}_{\alpha}$ in emission without clear evidence of P-Cyg profiles. The atmospheric parameters determined for TYC 6234-178-1 are $T_{\text {eff }}=19000 \mathrm{~K}, \log g=2.25 \mathrm{dex}$ and $\xi=14.0 \mathrm{~km} \mathrm{~s}^{-1}$. This object has the highest helium abundance $(+0.21 \mathrm{dex})$ amongst our sample and it shows a nitrogen enrichment of 0.3 dex relative to the Sun (Asplund et al. 2006). As discussed in Sect. 4.3.3, this abundance pattern could indicate the occurence of the second dredge-up or HBB, enhancing the helium and nitrogen abundance on the stellar surface. The silicon and sulfur abundances are similar to the mean abundances obtained for the whole sample, as can be seen in Fig. 10.

\section{LSS 4634 (IRAS 18023-3409)}

Vijapurkar \& Driling (1993) classified this source as a B9Ia+e2+ star. Parthasarathy et al. (2000) classified it as a B2IIIe hot post-AGB star based on low-resolution spectra. Gauba \& Parthasarathy (2003) noted a considerable circumstellar extinction based on UV(IUE) spectra and estimated an effective temperature $T_{\text {eff }}=20300 \mathrm{~K}$ and $\log g=3.0$ dex based on the spectral type.

The visual inspection of our observed spectrum reveals strong forbbiden emission lines of $\mathrm{Fe}$ II, weak nebular lines caused by $\mathrm{N}_{\mathrm{II}}$ and P-Cyg profiles in Balmer lines $\mathrm{H}_{\gamma}$ and $\mathrm{H}_{\beta}$. Our analysis of LSS 4634 indicates a considerable N enrichment (strong $\mathrm{N}_{\text {II }}$ features can be seen in Fig. 2) associated with high He abundance, which may result from a second dredge-up episode or HBB process ocurred in AGB phase.

\section{LS 3099 (IRAS 13266-5551)}

Parthasarathy et al. (2000) classified LS 3099 as a hot post-AGB star with spectral type B1Ibe. Suarez et al. (2006) 
confirmed this object as a post-AGB star in their low-resolution spectra study. Sarkar et al. (2005) determined chemical abundances for this star based on a high-resolution $(R \sim 30000)$ spectrum.

We notice some differences between our observed spectrum and the spectrum published by Sarkar et al. (2005) observed in June 2002, especially the variation in the strength of the Balmer lines and the change of P-Cyg profiles of helium lines. These variations may indicate the occurrence of rapid evolutionary changes of the central object or important physical processes occuring in the envelope.

Sarkar et al. (2005) used a full LTE treatment with the suite ATLAS, WIDTH9 and SYNSPEC to derive the atmospheric parameters and the chemical abundances for this star based on simultaneous fits of $\mathrm{C}_{\text {III }}, \mathrm{N}_{\text {III }}, \mathrm{O}_{\text {II }}$ and Fe III lines. The spectral coverage of their data did not allow using the ionization balance to determine $T_{\text {eff }}, \log g$ and $\xi$. The analysis of Sarkar et al. (2005) was limited to $\log g>3.0$ dex constrained by the model convergence. Therefore, it is likely that the obtained surface gravity is an upper limit. The best fit to the observed spectrum was obtained for $T_{\text {eff }}=23000 \mathrm{~K}, \log g=3.0 \mathrm{dex}$ and $\xi=10 \mathrm{~km} \mathrm{~s}^{-1}$. Their derived elemental abundances are 11.26 for helium, 8.67 for carbon, 8.23 for nitrogen, 8.78 for oxygen, and 7.96 for sulfur.

In contrast to their results, we found $T_{\text {eff }}=20200 \mathrm{~K}$, $\log g=2.38 \mathrm{dex}$ and $\xi=16.0 \mathrm{~km} \mathrm{~s}^{-1}$ in our analysis based on large coverage and a high-resolution spectrum. In addition, our results do not support the carbon enrichment pointed by Sarkar et al. (2005): our analysis indicates sub-solar abundance of carbon $([\mathrm{C} / \mathrm{H}]=-0.12 \mathrm{dex})$, and relevant nitrogen enrichment $([\mathrm{N} / \mathrm{H}]=+0.72 \mathrm{dex})$ for the star LS 3099.

\section{LSE 76 (IRAS 17311-4924)}

This source was identified for the first time as an emissionline star by Henize (1976). Kozok (1985) classified it as a Be star and derived a photometric distance to the Sun of $2.6 \mathrm{kpc}$. Several circumstellar studies of this source can be found in the literature. Van der Veen et al. (1989) modelled the circumstellar shell using visual and infrared observations and obtained a photometric distance of $2.5 \mathrm{kpc}$ to the Sun, a dust temperature of $124 \mathrm{~K}$ and temperature of $14000 \mathrm{~K}$ for the central star. LSE 76 was classified as a post-AGB star by Parthasarathy \& Pottasch (1989) based on far-infrared (IRAS) excess. Suarez et al. (2006) classified it as a transition object based on a low-resolution spectrum. Parthasarathy et al.(2000) classified it as a hot post-AGB star with spectral type B1Ile. Based on this spectral type, Gauba $\&$ Parthasarathy (2003) estimated the effective temperature and surface gravity of $20300 \mathrm{~K}$ and 3.0 dex, respectively. Sarkar et al. (2005) studied LSE 76 using high-resolution spectra with short spectral coverage: the few detected absorption lines did not allow the analysis of the stellar atmosphere.

The comparison between our observed spectra of LSE 76 and that obtained by Sarkar et al. (2005) reveals remarkable changes. We note a considerable increase in the emission flux of $\mathrm{H}_{\alpha}$ emission and variations in the emission component of P-Cyg profiles of He I lines. Some emission lines of Fe III and V I due to circumstellar material also show signs of variation.

We obtained $T_{\text {eff }}=20500 \mathrm{~K}, \log g=2.35 \mathrm{dex}$ and $\xi=$ $16.0 \mathrm{~km} \mathrm{~s}^{-1}$ for LSE 76. The present value of effective temperature agrees with that obtained by Sarkar et al. (2005). However, the derived values of surface gravity are very different in both studies. The radial velocity is also slightly different: we obtained a mean radial velocity of $42.30 \mathrm{~km} \mathrm{~s}^{-1}$ based on measurements of well-defined and unblended absorption lines, while Sarkar et al. (2005) obtained $27.55 \mathrm{~km} \mathrm{~s}^{-1}$. Despite this difference in the radial velocity, no other signs of binarity, such as doubled or asymmetric lines, are found in our spectrum.

\section{LS IV-12 111 (IRAS 19590-1249)}

This source is a well-known hot post-AGB star, originally classified as a young emission-line star by Wackerling (1970). Kilkenny \& Pauls (1990) confirmed its emission-line nature and indicated a spectral type of approximately B0, based on a low-dispersion spectrum. McCausland et al. (1992) proceeded an LTE abundance analysis based on a high-resolution spectrum and obtained anomalous chemical composition for this star, concluding that it is likely a post-AGB star. Conlon et al. (1993) added that LS IV-12 111 is a post-AGB star or an emerging young halo planetary nebula with a central star with mass of $0.67 M_{\odot}$ based on multiwavelenght observations. Ryans et al. (2003) determined the chemical composition of this source based on a non-LTE formalism and confirmed LS IV-12 111 as a post-AGB star or very young planetary nebula. Umana et al. (2004) detected radio emission around the nebula in the $8.4 \mathrm{GHz}$ with flux of $2.76 \mathrm{mJy}$ at $3.6 \mathrm{~cm}$ and suggested that this target is a transition object emerging as a young planetary nebula. Other works based on low-resolution spectra classified LS IV-12 111 as a post-AGB star (Parthasarathy et al. 2000) or transition object (Suarez et al. 2006).

Some studies, however, suggested that this star is likely a binary object. Photometric variations have been reported by Arkhipova et al. (2002), and Ryans et al. (2003) obtained a variation of $14 \mathrm{~km} \mathrm{~s}^{-1}$ in the radial velocities measured in three consecutive nights in 1994. However, the observed emission spectrum showed no evidence of time-dependent behaviour. De Marco et al. (2004) found a variability of $39 \mathrm{~km} \mathrm{~s}^{-1}$ in their radial velocity measurements in August 2002 and September 2003 and concluded that this star is actually a binary.

The heliocentric radial velocity determined for LS IV-12 111, $V_{r}=86.9 \mathrm{~km} \mathrm{~s}^{-1}$ (Table 1) is slightly different from the values previously listed in the literature. However, because we found no spectroscopic signs of binarity in our data, this star was analysed as a single star.

The atmospheric parameters determined for this source in our analysis are $T_{\text {eff }}=21000 \mathrm{~K}, \log g=2.42 \mathrm{dex}$ and $\xi=20.0 \mathrm{~km} \mathrm{~s}^{-1}$ and are consistent with the non-LTE analysis of Ryans et al. (2003). The chemical abundances obtained for LS IV-12 111 are similar to those obtained for the other objects of our sample, as shown in Fig. 10. In Fig. 11 we compare the chemical compositon derived here with the results of McCausland et al. (1992) and Ryans et al. (2003). Large differences in the carbon abundances may be caused by the approach in the analysis, the adopted carbon model atom and/or the linelist used to determine the mean abundance. These questions will be discussed in Sect. 4.3.3.

\section{LSE 63 (IRAS 18371-3159)}

This star is listed as an $\mathrm{OB}+$ star in the Case-Hamburg OB-star surveys (Drilling \& Bergeron 1995). Parthasarathy et al. (2000) detected $\mathrm{H}_{\beta}$ and $\mathrm{H}_{\gamma}$ in emission and classified LSE 63 as a hot post-AGB star based on far-infrared colours similar to PNe, high galactic latitude and B supergiant spectral type (B1Iabe). Umana et al. (2004) detected the radio emission of $0.62 \mathrm{mJy}$ in $3.6 \mathrm{~cm}$ at $8.4 \mathrm{GHz}$, confirming the post-AGB status with 


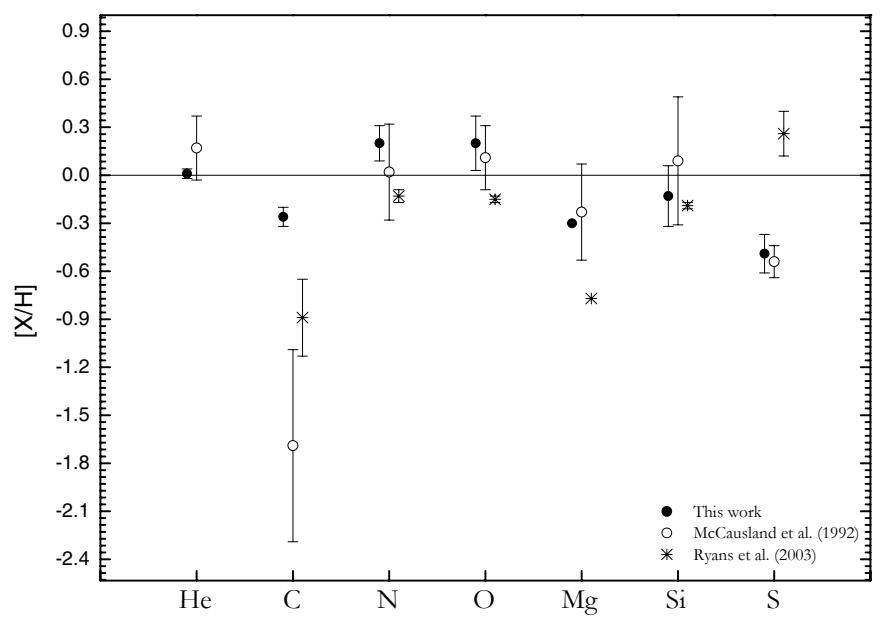

Fig. 11. Differential chemical abundances derived here for the hot postAGB star LS IV-12 111 (IRAS 19590-1249) compared with the previous results of McCausland et al. (1992) and Ryans et al. (2003). The black line shows the solar abundance reference of Asplund et al. (2006). The error bars in our study and in Ryans et al. (2003) represent standard deviations whilst it indicates the uncertainty in the abundance determination of McCausland et al. (1992).

the presence of a low-excitation nebula. Gauba \& Parthasarathy (2003) detected a low circumstellar extinction and, considering a spectral type of B1Iabe, they estimated the effective temperature and surface gravity to be $20800 \mathrm{~K}$ and 2.9 dex, respectively.

The observed spectrum for this source is very similar to that of LS IV-12 111, the only difference being a slightly higher projected rotational velocity for the former, as shown in Fig. 2. Several helium lines show P-Cyg profiles, and other signatures of stellar wind are also present in lines of hydrogen, iron, and carbon. Low-excitation nebular lines of $\left[\mathrm{N}\right.$ II] and $\left[\mathrm{S}_{\mathrm{II}}\right]$ are also present.

In our analysis, the atmospheric parameters determined for this object are $T_{\text {eff }}=21600 \mathrm{~K}, \log g=2.58 \mathrm{dex}$ and $\xi=$ $20.0 \mathrm{~km} \mathrm{~s}^{-1}$. Our abundance results reveal solar helium abundance and a low nitrogen enrichment. Determining the carbon abundance was not possible, since the lines commonly used in our analysis are in emission or affected by severe line blending. Furthermore, the $\mathrm{C}_{\text {II }}$ lines of the multiplets at 5132-5151 $\AA$ and 6779-6803 $\AA$ are not visible in the spectrum, suggesting a low carbon abundance.

\section{LSE 148 (HD 177566)}

This source was originally classified as a B1III star by Hill (1970) based on photoeletric observations during 1963-1964. Using the reddening-free colour index $\left[c_{1}\right]$, Keenan \& Dufton (1983) derived an effective temperature and surface gravity of $31000 \mathrm{~K}$ and 3.5 dex, respectively. Kendall et al. (1994) derived atmospheric parameters and LTE chemical abundances based on high-resolution optical spectrum and UV(IUE) data.

LSE 148 is a very intriguing object and a quick glance at Fig. 2 shows the scarcity of metal lines in the observed spectrum. There are few relatively strong absorption lines in its spectrum, all of them caused by hydrogen and helium. Unlike other stars of our sample, no emission line was identified for LSE 148. Kendall et al. (1994) also reported this lack of emission lines in the spectrum obtained in September 1993. However, these authors discussed a possible trace of emission in the $\mathrm{H}_{\beta}$ profile due to inconsistencies in measurements of surface gravity by different methods. In our spectrum, we detected a flattened shape in the cores of the $\mathrm{H}_{\alpha}$ and $\mathrm{H}_{\beta}$ profiles, which may indicate the presence of weak emission. Kendall et al. (1994) detected the lines $\mathrm{N}_{\text {II }} 3995 \AA$ and $\mathrm{C}_{\text {III }} 4647 \AA$ in their spectral analysis, however, in our spectra, we did not detect the carbon line and the nitrogen line is absent or very weak. On the other hand, we detected some $\mathrm{N}$ III lines not mentioned by the former authors, such as N III $4640 \AA$ and N III $4642 \AA$. Measurements of equivalent widths for some lines in common with Kendall et al. (1994) were compared and we found an offset of $\sim 10 \mathrm{~m} \AA$ in the measurements, our values being higher than those of Kendall et al. (1994).

The atmospheric parameters and chemical abundances derived by Kendall et al. (1994) were determined by LTE synthesis. The effective temperature $\left(T_{\text {eff }}=30000 \mathrm{~K}\right)$ was obtained by $\mathrm{He}_{\mathrm{I}} / \mathrm{II}$ ionization balance and the surface gravity $(\log g=$ $3.8 \mathrm{dex}$ ) was estimated by fitting theoretical profiles to the normalized $\mathrm{H}_{\delta}$ line. They adopted a fixed value of $5 \mathrm{~km} \mathrm{~s}^{-1}$ for the microturbulent velocity. We obtain $T_{\text {eff }}=30900 \mathrm{~K}, \log g=$ 3.83 dex and $\xi=3.0 \mathrm{~km} \mathrm{~s}^{-1}$ for this object, in good agreement with Kendall et al. (1994). Our derived abundances are consistent with the abundances obtained by Kendall et al. (1994) (except for nitrogen: we obtained $\log \epsilon(\mathrm{N})=7.23$ while Kendall et al. (1994) derived an upper limit of 6.9 dex), confirming that the LSE 148 is a metal-poor star.

In their work, Kendall et al. (1994) estimated a mass of $0.55 M_{\odot}$ for LSE 148 , based on evolutionary tracks according to Schönberner $(1983,1987)$ for post-AGB stars. Combining this fact and the lack of detected nebulosity around the star, the authors discussed the possibility that this object may be slowly evolving into the white dwarf region in the HR diagram, though it has not reached the planetary nebulae phase yet. We will discuss the evolution status of this object in Sect. 4.3.4.

\subsection{Abundances}

Recent abundance analyses of post-AGB stars reported in the literature reveal a complex scenario. In his review, Van Winckel (2003) pointed out the main chemical characteristics of postAGB and candidates, especially the optically bright F-G type post-AGB objects. According to this author, hot post-AGB have been detected with low metal abundances, especially carbon, approximately solar helium abundance and, in some cases, a possible nitrogen enrichment. From our analysis, the obtained abundance pattern indicates that these evolved objects had their surface abundances changed during the previous AGB phase.

\subsubsection{The $\log \mathrm{C} / \mathrm{N}-\log \mathrm{O} / \mathrm{N}$ diagram}

The mass estimates play an important role in the discussion of the evolutionary scenario and the chemical abundances obtained in our analysis. The $\mathrm{C} / \mathrm{O}$ ratio is commonly used to separate the carbon-rich and carbon-poor objects. The third dredge-up process is supposed to be responsible for the carbon enrichment (Iben \& Renzini 1983). In Fig. 12, the objects of our sample are located in the $\log (\mathrm{C} / \mathrm{N}) \times \log (\mathrm{O} / \mathrm{N})$ diagram. In the same figure we also display abundance ratios for several classes of objects such as Galactic carbon stars (Lambert et al. 1986), some cool post-AGB stars (Luck et al. 1990; Van Winckel 1997), the PNe samples of Kingsbrugh \& Barlow (1994) and Peimbert \& Torres-Peimbert (1983). Our sample stars present a carbon-poor pattern and follow the same behaviour as that of the PNe sample. 


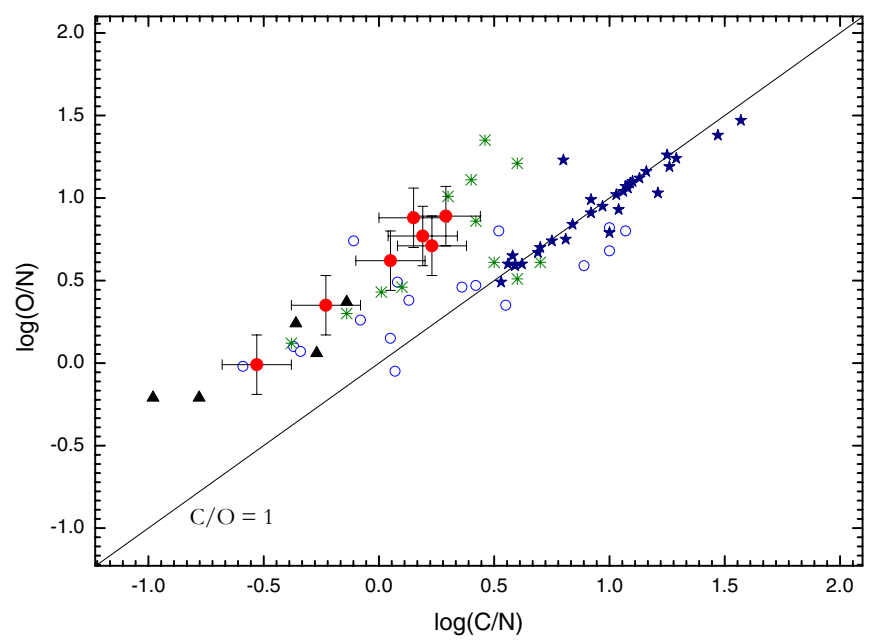

Fig. 12. Relative abundances $\log (\mathrm{O} / \mathrm{N}) \times \log (\mathrm{C} / \mathrm{N})$ for several classes of stars. Results for type I PNe (black filled triangles), type II PNe (green asterisks), cool post-AGB stars (blue open circles), Galactic carbon stars (blue filled stars) can be compared to the hot post-AGB stars analysed in this work (red filled circles). The solid line indicates $\mathrm{C} / \mathrm{O}=1$.

\subsubsection{Oxygen and the $\alpha$-elements}

Low and high oxygen abundances have been reported in the literature for hot post-AGB stars (McCausland et al. 1992). Because our objects are on the left side of the solid line $(\mathrm{C} / \mathrm{O}=1)$ in Fig. 12, they can be considered to be oxygen-rich. Similar results for LSE 3, TYC 6234-178-1 were reported by Cerrigone et al. (2009) based on the detection of silicate features at $10 \mu \mathrm{m}$ typical of circumstellar O-rich environments. For LSE 63 and LS IV-12 111, the same authors detected a dual-chemistry circumstellar envelope, associated with the $10 \mu \mathrm{m}$ feature and silicate features due to PAHs.

The analysis of the $\alpha$-elements reveals a different scenario. Low abundances for magnesium and silicon have been reported in the literature. The published results for sulfur indicate either a lack or an enrichment of this element (Van Winckel 2003). Our results show roughly solar abundances for $\mathrm{Mg}$ and $\mathrm{Si}$ (excluding LSE 148), with considerable scatter. We obtained an average abundance of 6.77 for suphur, with values varying from 6.57 to 7.11 dex.

\subsubsection{Carbon and nitrogen}

Previous works suggested that carbon is notably underabundant in hot post-AGB stars. As extreme examples, Moehler \& Heber (1998) found $[\mathrm{C} / \mathrm{H}]=-2.23$ dex for PG 1323-086 and Conlon et al. (1991) found $[\mathrm{C} / \mathrm{H}]=-2.3$ dex for PHL 174. However, these results are based mostly on the LTE analyses of $\mathrm{C}_{\text {II }}$ lines at $6578 \AA$, $6582 \AA$, and especially at $4267 \AA$, which generally suffer significant non-LTE effects (McErlean et al. 1999; Nieva \& Przybilla 2008).

In Fig. 13 we compare the distribution of carbon abundances obtained in this work with other results from the literature for several cool (Stasińska et al. 2006) and hot post-AGB stars, with some objects in common. We observed a large difference between the carbon abundances obtained for cool and hot post-AGB stars collected from the literature. Our results do not show strong underabundances of carbon, as reported in previous works.

An excess of nitrogen relative to the sun was detected in all objects of our sample, except for LSE 148. Some results
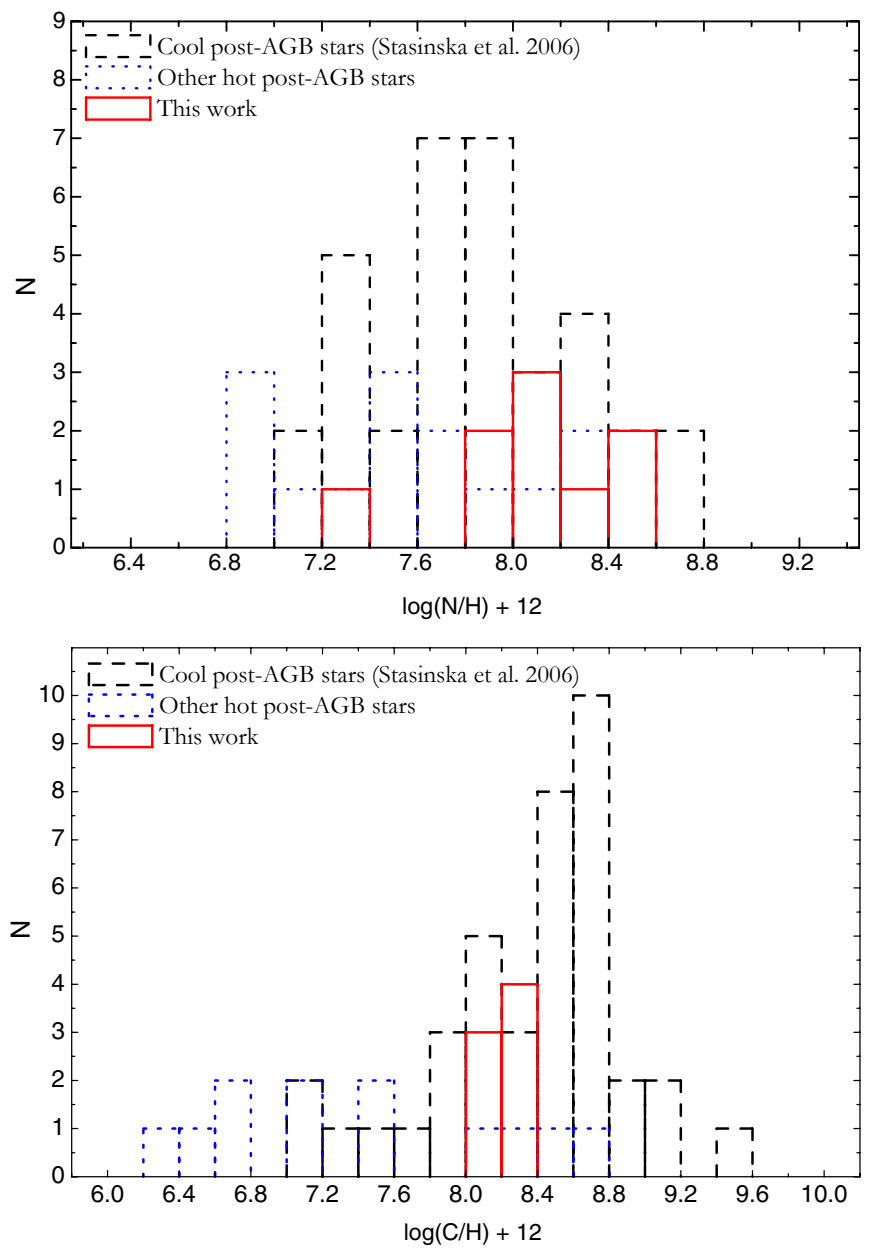

Fig. 13. Comparison between the distributions of the obtained carbon (bottom) and nitrogen (top) abundances and other results for cool and hot post-AGB stars (Moehler \& Heber 1998; McCausland et al. 1992; Sarkar et al. 2005; Ryans et al. 2003; Hambly et al. 1996; Kendall et al. 1994; and Conlon et al. 1991).

for the literature show nitrogen enrichment in optically bright F-G type post-AGB stars (see for example Sivarani et al. 1999 and Van Winckel 1997) as well as some hot post-AGB. For example, the high-latitude star $\mathrm{CPD}-61^{\circ} 455$ displays $[\mathrm{N} / \mathrm{H}]=$ +0.38 dex, as detected by Hambly et al. (1996). On the other hand, underabundance of nitrogen has been obtained for other hot post-AGB objects (Moehler \& Heber 1998). Therefore, it seems that the dredge-up of $\mathrm{CNO}$ processed material may have occurred for several objects in this evolutionary phase. In particular, we note the nitrogen enrichment for two objects of our sample, LSS 4634 and LS 3099, located on the bottom left of Fig. 12. In Fig. 13 we compare the distribution of carbon and nitrogen abundances obtained in this work with other results from the literature for several cool (Stasińska et al. 2006) and hot postAGB stars, with some objects in common. We observed a large difference between the carbon abundances obtained for cool and hot post-AGB stars collected from the literature and a wide variation in the nitrogen abundances obtained by several analyses. Our results do not show strong underabundances of carbon, as reported in previous works.

We can invoke some mixing processes in the AGB phase that may be responsible for these results. The occurrence of the third dredge-up in the AGB phase is directly related to the high abundance of carbon. This mixing episode occurs when the convective envelope penetrates into the interface region between the $\mathrm{H}$ 
and He burning layers during the thermal pulse and thus, the processed carbon is dredged into the stellar photosphere (Iben \& Renzini 1983). The efficiency of the dredge-up process is related to the core mass of the object, the mass of dredged material to the surface, and the stellar metallicity. The third dredgeup is expected to achieve maximum efficiency in stars with masses in the range 1.2 to $3.0 M_{\odot}$ (García-Lario 2006) and to decrease with increasing metallicity (Karakas et al. 2002). Other consequences of the third dredge-up are the photospheric enrichment with $s$-process elements, detected in several cool post-AGB stars (Van Winckel 2003). However, no evidence of $s$-process elements was detected in our observed spectra.

Another possibility to explain the enrichment of nitrogen and helium is the occurrence of the second dredge-up. This mixing process occurs for intermediate-mass stars with $M \geq 4 M_{\odot}$ (depending on the chemical composition) when the object is ascending to the AGB after the exhaustion of the helium core. At this stage, the expansion of the outer layer extinguishes the hydrogen burning shell, which causes the convective envelope to move inward for the second time. This second dredge-up brings the hydrogen burning products, mainly helium and nitrogen $\left({ }^{4} \mathrm{He}\right.$ and $\left.{ }^{14} \mathrm{~N}\right)$ to the surface, whilst the amount of ${ }^{12} \mathrm{C}$ and ${ }^{16} \mathrm{O}$ decreases (Boothroyd \& Sackmann 1999; Kwok 2000).

The occurrence of "hot bottom-burning" (HBB) also could possibly explain the oxygen enrichment at the expense of carbon. During the thermal pulsing phase, intermediate-mass stars (3.5 $M_{\odot} \leq M \leq 5-8 M_{\odot}$ ) with solar metallicity experience a nuclear H-burning that extends from the radiative shell into the deepest layer of the convective envelope during the inter-pulse periods. The main nuclear products are due to $\mathrm{CNO}$ cycle, so that it is expected that the surface abundances of $\mathrm{He}$ and $\mathrm{N}$ are increased. The ${ }^{12} \mathrm{C}$ is processed into ${ }^{13} \mathrm{C}$ and ${ }^{14} \mathrm{~N}$ and thus the $\mathrm{C} / \mathrm{O}$ remains $<1$, preventing the carbon enrichment. Other products of the $\mathrm{HBB}$ can also be brought to the surface, such as isotopes of $\mathrm{Ne}, \mathrm{Ca}, \mathrm{Mg}$, and $\mathrm{Al}$ as well as ${ }^{7} \mathrm{Li}$, through the Cameron-Fowler mechanism (Lattanzio 2003). However, models have shown that the dependence on metallicity should not be ignored because it provides the actual chemical changes due to HBB (García-Hernández et al. 2006).

In our analysis, the HBB could explain not only the carbon underabundance, but aditionally the enrichment of nitrogen for some objects in our sample. The type I PNe are classified based on their abundances of He and N, namely, $(\mathrm{He} / \mathrm{H})>0.14$ or $\log (\mathrm{N} / \mathrm{O})>0$ (Peimbert \& Torres-Peimbert 1983). It is believed that they are originated from the intermediate-mass stars like the objects in our sample. LSS 4634 is the only star in our sample that has the characteristics as defined by Peimbert \& Torres-Peimbert (1983), and therefore, is a possible candidate to form a PNe type I in the future, as can be seen in Fig. 12. Another important point is presented in Fig. 14, which shows the clear relation between $\mathrm{He}$ and $\mathrm{N}$ enrichment obtained for our stars, excluding LSE 148. This relation suggests the probable occurrence of HBB for the objects of our sample. The HBB has also been suggested as the most likely mechanism to explain the formation of PNe type I (Marigo 2007) and the chemical abundances of massive O-rich AGB stars, called OH/IR stars (García-Hernández et al. 2006).

The obtained abundances combined with the $M_{\text {ZAMS }}$ and metallicity estimates for our objects suggest that the HBB and/or the second dredge-up might have ocurred in the AGB phase (except for LSE 3 and LSE 148). However, considering the uncertainties in the mass estimates and their location close to the minimal threshold where the HBB possibly begins to take place (according to evolutionary models), it is not ruled out that



Fig. 14. Position of massive post-AGB stars in our sample in the $(\mathrm{He} / \mathrm{H}) \times(\mathrm{N} / \mathrm{H})$ plane indicating a possible relationship between the $\mathrm{He}$ and $\mathrm{N}$ enrichments.

the third dredge-up could have worked at any moment of the AGB phase, although with less efficiency. Indeed, depending on the mass loss in the AGB phase during the thermal pulses, the convective envelope can become less massive and eventually the HBB ceases to operate when the temperatures declines. In this case, the third dredge-up can bring some dredged ${ }^{12} \mathrm{C}$ to the surface, even for a short time (Vassiliadis \& Wood 1993). Moreover, if the HBB had occurred, it would have been more efficient for some objects such as LSS 4634, based on the obtained abundance ratios.

\subsubsection{LSE 148}

The analysis of LSE 148 suggests it is a metal-poor, low-mass star $\left(M_{\text {ZAMS }} \sim 1-2 M_{\odot}\right)$. The scarcity and relative strength of metal lines, as shown in Fig. 2, is a first evidence of low metallicity. Unfortunately, no iron lines were identified in its spectrum from which to estimate the metallicity. However, the metallicity based on CNO abundances indicates a very low value $(Z=0.001)$. Lower massive objects like LSE 148 can spend $10^{4}$ to $10^{5}$ years in the post-AGB phase (Schönberner 1979a,b). If LSE 148 is indeed a post-AGB star, its circumstellar envelope might have been dissipated in its post-AGB track, resulting in the absence of emission profiles in the observed spectrum.

LSE 148 could also be explained as an O-type hot subdwarf (sdO). According to Heber (2009), the hot subdwarf stars ( $\mathrm{sdB}, \mathrm{sdOB}$ or $\mathrm{sdO})$ are evolved objects with luminosity ranging from $10-10^{3} L_{\odot}$, and high values of surface gravity between 5.0 and 6.0 dex, which may or may not present a He-rich spectrum. Some sdO stars evolving directly from the AGB may present $\log g$ values lower than those of typical sdOBs evolved from the extreme horizontal branch (Heber 1986). Dorman et al. (1993) discussed the evolution from the BHB and EHB and showed that some objects may evolve directly to white dwarf (AGB manqué stars) or ascend to the AGB, without going through thermal pulses (post-early AGB stars). In Fig. 9, we show LSE 148 and the other sample stars in the $\log T_{\text {eff }}-\log g$ diagram, with the post-HB evolutinary tracks according to Dorman et al. (1993) computed for core masses of $0.49,0.5,0.51$, and $0.53 M_{\odot}$. We also show some sdOB stars from Heber (1986) and Ahmad \& Jeffery (2003) for comparison. The location of LSE 148 in Fig. 9 suggests that LSE 148 may be a post-HB star. A definite classification should consider additional criteria such as 
luminosity and surface gravity. Using the relation of Wood \& Zarro (1981) and the mass obtained for this object, we estimate $\log L / L_{\odot}=3.5$ for LSE 148, just above the threshold value for the subdwarfs. The $\log g$ value obtained for LSE 148 (3.83 dex) is not consistent with the typical $\log g$ for hot subdwarfs (however, some sdO stars such as the object KS 292 (Rauch et al. 1991) may show some parameters similar to those of LSE 148). For LSE 148, the hypothesis of post-early AGB status is more reasonable, since the non-occurrence of the thermal pulses in the AGB phase and the lack of nebulosity around the central object may explain the relatively low surface gravity and the observed abundance pattern.

\section{Summary and conclusions}

We derived chemical abundances of $\mathrm{He}, \mathrm{C}, \mathrm{N}, \mathrm{O}, \mathrm{Mg}, \mathrm{Si}$, and $\mathrm{S}$ for a sample of nine hot post-AGB stars based on non-LTE analysis of high-resolution optical spectra. The main conclusions of this paper are summarized as follows:

1. The object SAO 209396 shows direct observational evidence of being a double-lined spectroscopic binary (SB2) and therefore, we did not perform the abundance analysis for this object. The status of the object is still discussed in the literature.

2. All objects analysed in this work show emission lines in their spectra mainly caused by hydrogen, helium, silicon, and iron except for LSE 148, which presents flattened cores of $\mathrm{H}_{\alpha}$ and $\mathrm{H}_{\beta}$ profiles. Additionaly, this object shows few and weak metal lines in the observed absorption spectrum, suggesting low metallicity. The P-Cyg profiles are also detected for several objects mainly due to hydrogen, indicating ongoing mass loss for these hot post-AGB stars.

3. Our analysis indicates a underabundance of carbon, while helium has a slight over-abundance for some objects. Nitrogen appears over-abundant for most objects of the sample. Although the abundance pattern indicates low values for carbon (relative to the solar value), the analysis presented here is not consistent with previous analyses that have reported strong carbon depletions. Previous analyses in LTE formalism are likely to be the source of this discrepancy. The obtained $\mathrm{C} / \mathrm{O}$ ratio for the objects is consistent with O-rich circumstelar dust detected for some objects of our sample reported by Cerrigone et al. (2009) based on infrared observations with the Spitzer Space Telescope and previous radio observations.

4. The sample stars were placed on $\log T_{\text {eff }}-\log g$ diagram and, based on evolutionary tracks of post-AGB stars (Schönberner 1983; Blöcker 1995), the core masses were estimated. Except for LSE 148 whith $M=0.55 M_{\odot}$ (it seems that this is a post-early AGB star), the masses for the other stars vary from 0.6 to $0.84 M_{\odot}$. These objects are likely to be high-mass post-AGB stars rapidly evolving towards the PNe phase. Indeed, some nebular lines (for example, $[\mathrm{N} \mathrm{II}]$ and $\left[\mathrm{S}_{\mathrm{II}}\right]$ ) were detected in the spectra of most of the objects of our sample, indicating that the photo-ionization of the circumstellar envelope has been started recently.

5. Nitrogen enrichment (relative to the Sun) is relatively high for two objects of our sample: LS $3099(+0.72$ dex $)$ and LSS 4634 (+0.81 dex). The latter object has chemical characteristics similar to type I PNe as defined by Peimbert \& Torres-Peimbert (1983) and is therefore the likely candidate to form a type I PNe.
6. The detected underabundance of carbon can be explained by the absence of the third dredge-up episode. On the other hand, the high nitrogen abundance correlates with a slight excess of the helium for several objects and the high estimated masses for the sample objects seem to indicate the possible occurrence of the HBB process in the previous AGB phase. The effect of this process is possibly different for each object, being more efficient for the most massive stars and for those objects whose third dredged-up was less efficient.

Acknowledgements. We are grateful to the referee for rich comments and suggestions. D.R.C.M. thanks CAPES (Coordenação de Aperfeiçoamento de Pessoal de Nível Superior) for a Ph.D. fellowship and the Graduate Program of the Observatório Nacional for financial support during the observing runs at ESO.

\section{References}

Acker, A., Ochsenbein, F., Stenholm, B., et al. 1992, Strasbourg-ESO Catalogue of Galactic Planetary Nebulae, ESO, Garching

Ahmad, A., \& Jeffery, C. S. 2003, A\&A, 402, 335

Arkhipova, V., Ikonnikova, N., Noskova, P., \& Komissarova, G. 2002, Astron. Lett., 28, 257

Asplund, M., Grevesse, N., \& Sauval, A. 2006, Nucl. Phys. A, 777, 1

Blöcker, T. 1995, A\&A, 299, 755

Boothroyd, A., \& Sackmann, I.-J. 1999, ApJ, 510, 232

Cantiello, A., Langer, N., Brott, I., et al. 2009, A\&A, 499, 279

Cerrigone, L., Hora, J., Umana, G., \& Trigilio, C. 2009, ApJ, 703, 585

Conlon, E., Dufton, P., Keenan, F., \& McCausland, R. 1991, MNRAS, 248, 820 Conlon, E., Dufton, P., Keenan, F., McCaulsland, R., \& Holmgren, D. 1992, ApJ, 400, 273

Conlon, E., Dufton, P., McCaulsland, R., \& Keenan, F. 1993, ApJ, 408, 593

Crampton, D. 1971, AJ, 76, 260

Crampton, D. 1972, MNRAS, 158, 85

De Marco, O., Bond, H., Harmer, D., \& Fleming, A. 2004, ApJ, 602, L93

de Wit, W., Testi, L., Palla, F., \& Zinnecker, H. 2005, A\&A, 437, 247

Dorman, B., Rood, R. T., \& O'Connell, R. W. 1993, ApJ, 419, 596

Downes, R., \& Keyes, C. 1988, AJ, 96, 777

Drilling, J., \& Bergeron, L. 1995, PASP, 107, 846

García-Hernández, D., García-Lario, P., Plez, B., Manchado, A., \& D’Antona, F. 2006, Planetary Nebulae in our Galaxy and Beyond, ed. M. J. Barlow, \& R. H. Méndez (Cambridge University Press), IAU Symp., 234, 87

García-Lario, P. 2006, Planetary Nebulae in our Galaxy and Beyond, ed. M. J. Barlow, \& R. H. Méndez (Cambridge University Press), IAU Symp., 234, $63 \mathrm{G}$

García-Lario, P., Manchado, A., Pych, W., \& Pottasch, S. 1997, A\&AS, 126, 479

Gauba, G., \& Parthasarathy, M. 2003, A\&A, 407, 1007

Gies, D. 1987, ApJS, 64, 545

Gies, D., \& Lambert, D. 1992, ApJ, 387, 673

Greenstein, J., \& Sargent, A. 1974, ApJS, 28, 157

Hambly, N., Dufton, P., Keenan, F., \& Lumsden, L. 1997, MNRAS, 278, 811

Heber, U. 1986, A\&A, 155, 33

Heber, U. 2009, ARA\&A, 47, 211

Henize, K. 1976, ApJS, 30, 491

Herwig, F. 2005, ARA\&A, 43, 435

Hill, P. W. 1970, MNRAS, 150, 23

Hrivnak, B. 1997, IAUS, 180, 303

Hubeny, I., \& Lanz, T. 1995, ApJ, 439, 875

Iben, I., \& Renzini, A. 1983, ARA\&A, 21, 271

Karakas, A., Lattanzio, J., \& Pols, O. 2002, PASA, 19, 515

Kaufer, A., Stahl, O., Tubessing, S., et al. 1999, The Messenger, 95, 8

Kendall, T., Brown, P., Conlon, E., Dufton, P., \& Keenan, F. 1994, A\&A, 291, 851

Keenan, F., \& Dufton, P. 1983, MNRAS, 205, 435

Kilkenny, D., \& Pauls, L. 1990, MNRAS, 244, 133

Kingsbrugh, R., \& Barlow, M. 1994, MNRAS, 271, 257

Kozok, J. 1985, A\&AS, 62, 7

Kurucz, R. 1994, Solar Abundance Model Atmospheres, CDROM No. 19, Smithsoniam Astrophysical Observatory

Kwok, S. 2000, The Origin and evolution of Planetary Nebulae (Cambridge University Press)

Lambert, D., Gustafsson, B., Eriksson, K., \& Hinkle, K. 1986, ApJS, 62, L373

Lang, K. 1992, Astrophysical Data: Planets and Stars (Springer-Verlag) 
Lanz, T. \& Hudeny, I. 2003, ApJS, 146, 417

Lanz, T., \& Hudeny, I. 2007, ApJS, 169, 83

Lattanzio, J. 2003, 3D Stellar Evolution, ASPC, 293, L180

Luck., R., Bond, H., \& Lambert, D. 1990, ApJ, 357, L188

Magee, H., Dufton, P., Keenan, F., et al. 2001, MNRAS, 324, 747

Marigo, P. 2007, A\&A 467, 1139

Martin, J. 2004, ApJ, 128, 2474

McCausland, R., Conlon, E., Dufton, P., \& Keenan, F. 1992, ApJ, 394, 298

McErlean, N., Lennon, D., \& Dufton, P. 1999, A\&A, 349, 553

Méndez, R., Kudritzki, R., Herrero, A., Hulsfel, D., \& Groth, H. 1988, A\&A, 190, 113

Moehler, S., \& Heber, U. 1998, A\&A, 335, 985

Nieva, M., \& Przybilla, N. 2008, A\&A, 481, 199

Oudmaijer, R. 1996, A\&A, 306, 823

Parthasarathy, M. 1993, in Luminous High-Latitude Stars, ed. D. D. Sasselov

(San Francisco, CA: ASP), ASP Conf. Ser., 45, 173

Parthasarathy, M., \& Pottasch, S. 1989, A\&A, 225, 521

Parthasarathy, M., García-Lario, P., Pottasch, S., et al. 1993, A\&A, 267, L19

Parthasarathy, M., Vijapurkar, J., \& Driling, J. 2000, A\&AS, 145, 269

Peimbert, M., \& Torres-Peimbert, S. 1983, IAUS, 103, 233

Pereira, C. B., \& Miranda, L. F. 2007a, A\&A, 462, 231

Pereira, C. B., \& Miranda, L. F. 2007b, A\&A, 467, 1249

Perinotto, M., Morbidelli, L., \& Scatarzi, A. 2004, MNRAS, 349, 793

Pottasch, S., Olling, R., Bignell, C., \& Zijlstra, A. 1988, A\&A, 205, 248

Ratag, M., Pottasch, S., Zijlstra, A., \& Menzies, J. 1990, A\&A, 233, 181

Rauch, T., Heber, U., Hunger, K., Werner, K., \& Neckel, T. 1991, A\&A, 241, 457

Reyniers, M., Van de Steene, G., Van Hoof, P., \& Van Winckel, H. 2007, A\&A, 471, 247

Sarkar, G., Parthasarathy, M., \& Reddy, B. 2005, A\&A, 431, 1007
Schönberner, D. 1979a, A\&A, 79, 108

Schönberner, D. 1979b, ApJ, 272, 708

Schönberner, D. 1987, in Late Stages of Stellar Evolution, ed. S. Kwok, \& S. Pottasch (Dordrecht: Reidel), 359

Sharpless, S. 1953, ApJ, 118, 362

Simón-Díaz, S. 2010, A\&A, 510, A22

Sivarani, T., Parthasarathy, M., García-Lario, P., Manchado, A., \& Pottasch, S. 1999, A\&AS, 137, 505

Stasińska, G., Szczerba, R., Schmidt, M., \& Siódmiak, N. 2006, A\&A, 450, 701

Stephenson, C., \& Sanduleak, N. 1977, ApJS, 33, 459

Suárez, O., García-Lario, P., Manchado, A., et al. 2006, A\&A, 458, 173

Szczerba, R., Siódmiak, N., Stasińska, G., \& Borkowski, J. 2007, A\&A, 469, 799

Thé, P., De Winter, D., \& Pérez, M. 1994, A\&AS, 104, 315

Trundle, C., Dufton, P., Hunter, I., et al. 2007, A\&A, 471, 625

Umana, G., Cerrigone, L., Trigilio, C., \& Zappalà, R. 2004, A\&A, 428, 121

Van de Steene, G., Jacoby, G., \& Pottasch, S. 1996, A\&AS, 118, 243

Van der Veen, W., \& Habing, H. 1988, A\&A, 194, 125

Van der Veen, W., Habing, H., \& Geballe, T. 1989, A\&A, 226, 108

Van Winckel, H. 1997, A\&A, 319, 561

Van Winckel, H. 2003, ARA\&A, 41, 391

Vassiliadis, E., \& Wood, P. 1993, ApJ, 413, 641

Venn, K., Smartt, S., Lennon, D., \& Dufton, P. 1998, A\&A, 34, 987

Vijapurkar, J., \& Driling, J. 1993, ApJS, 89, 293

Wackerling, L. 1970, MNRAS, 73, 153

Waelkens, C., \& Waters, L. 2004, in Asymptotic Giant Branch Stars, ed. H. J. Habing, \& H. Olofsson (New York: Springer-Verlag)

Weidemann, V. 1987, A\&A, 188, 74

Weidemann, V. 2000, A\&A, 363, 647

Wood, P., \& Zarro, D. 1981, ApJ, 247, 247 